\title{
Bulk Rotational Symmetry Breaking in Kondo Insulator $\mathrm{SmB}_{6}$
}

\author{
Z. Xiang, ${ }^{1}$ B. Lawson, ${ }^{1}$ T. Asaba, ${ }^{1}$ C. Tinsman, ${ }^{1}$ Lu Chen,${ }^{1}$ C. Shang, ${ }^{2}$ X. H. Chen, ${ }^{2}$ and $\mathrm{Lu} \mathrm{Li}{ }^{1, *}$ \\ ${ }^{1}$ Department of Physics, University of Michigan, Ann Arbor, Michigan 48109, USA \\ ${ }^{2}$ Hefei National Laboratory for Physical Sciences at Microscale and Department of Physics, \\ University of Science and Technology of China, Hefei, Anhui 230026, China \\ and Key Laboratory of Strongly-coupled Quantum Matter Physics, \\ Chinese Academy of Sciences, Hefei, Anhui 230026, China \\ (Received 12 May 2017; revised manuscript received 10 July 2017; published 25 September 2017)
}

\begin{abstract}
The Kondo insulator samarium hexaboride $\left(\mathrm{SmB}_{6}\right)$ has been intensely studied in recent years as a potential candidate of a strongly correlated topological insulator. One of the most exciting phenomena observed in $\mathrm{SmB}_{6}$ is the clear quantum oscillations appearing in magnetic torque at a low temperature despite the insulating behavior in resistance. These quantum oscillations show multiple frequencies and varied effective masses. The origin of quantum oscillation is, however, still under debate with evidence of both twodimensional Fermi surfaces and three-dimensional Fermi surfaces. Here, we carry out angle-resolved torque magnetometry measurements in a magnetic field up to $45 \mathrm{~T}$ and a temperature range down to $40 \mathrm{mK}$. With the magnetic field rotated in the (010) plane, the quantum oscillation frequency of the strongest oscillation branch shows a fourfold rotational symmetry. However, in the angular dependence of the amplitude of the same branch, this fourfold symmetry is broken and, instead, a twofold symmetry shows up, which is consistent with the prediction of a two-dimensional Lifshitz-Kosevich model. No deviation of LifshitzKosevich behavior is observed down to $40 \mathrm{mK}$. Our results suggest the existence of multiple light-mass surface states in $\mathrm{SmB}_{6}$, with their mobility significantly depending on the surface disorder level.
\end{abstract}

DOI: 10.1103/PhysRevX.7.031054

In Kondo insulators, the physics is controlled by the strong many-body interactions [1]. The hybridization between the localized $f$ elections and conduction $d$ electrons causes the formation of Kondo singlets, which leads to a quench of local-magnetic-moment characteristics. Also, a narrow hybridization gap is developed at low temperature, resulting in a crossover from metallic to insulating behavior. In recent years, topological nontriviality is suggested to be hosted by Kondo insulators $[2,3]$. The opposite parity in the $f$ band (odd) and $d$ band (even) protects a band inversion similar to that in normal $Z_{2}$ topological insulators. In particular, the very large spin-orbit coupling in the renormalized $f$ electrons can give a system ground state with "nontrivial" topological order, i.e., a different topological invariant from that in vacuum. As a result, a gapless two-dimensional (2D) Dirac electron state, known as the topological surface state, has to exist at certain high-symmetry points in the surface Brillouin zone. Such predictions point out the Kondo insulators as promising candidates of interaction-driven topological

\footnotetext{
*Corresponding author. luli@umich.edu

Published by the American Physical Society under the terms of the Creative Commons Attribution 4.0 International license. Further distribution of this work must maintain attribution to the author(s) and the published article's title, journal citation, and DOI.
}

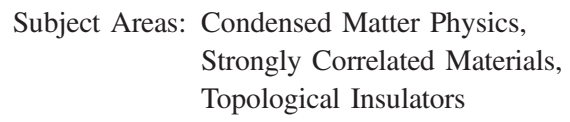

insulators, subsequently make this family a focus of attention in condensed-matter physics.

The cubic structured $\mathrm{SmB}_{6}$, the very first confirmed member of Kondo insulators [4], has been elaborately studied as the most feasible example of the electron-correlated threedimensional (3D) strong topological insulator [5-7]. A large amount of experimental observations on this material have been published [8], with some giving hints of the topological surface state [9-11], though the decisive evidence is yet to be found. The most striking discovery in $\mathrm{SmB}_{6}$ is the complicated de Haas-van Alphen ( $\mathrm{dHvA}$ ) oscillations detected at low temperature where the resistivity shows insulating behavior followed by a plateau below $3.5 \mathrm{~K}$. While our group has reported quantum oscillations corresponding to the 2D Fermi surface (FS) and light carriers that are consistent with the expectation on a typical topological surface state in aluminum-flux-grown samples [12], another work based on the floating-zone (FZ)-grown sample claimed the oscillations have 3D characters thus bulk origin, and an abnormally enhanced quantum oscillation amplitude suggesting a deviation from the Lifshitz-Kosevich (LK) theory below ${ }^{3} \mathrm{He}$ temperature [13]. To make it more confusing, no quantum oscillations have ever been observed in transport measurements $[12,14,15]$. Several theories have been proposed to reconcile the puzzling experimental results [16-18] as well as to explain the enriched exotic low-temperature behaviors in $\mathrm{SmB}_{6}$ [19-24]. The key problem to the 
confusion lies in the lack of a controlled study of the quantum oscillation amplitude.

In this work, we resolve the problem by mapping the oscillation amplitudes of different surfaces of the same crystal. We carry down magnetic torque measurements of flux-grown $\mathrm{SmB}_{6}$ single crystals [25] down to $40 \mathrm{mK}$ in a rotating magnetic field up to $45 \mathrm{~T}$. Our result shows a broken rotating symmetry in the amplitude of the main $\mathrm{dHvA}$ oscillation branch, indicating a 2D nature of the electronic state. In addition, neither very-high-frequency oscillations with 3D behavior nor abruptly enhanced dHvA amplitude suggesting a failed LK description is observed in any of our samples. These observations point to multiple 2D metallic states with small effective mass existing in the Kondo insulator $\mathrm{SmB}_{6}$.

By using the capacitive magnetic torque magnetometer shown in Fig. 1(a), we observe clear dHvA oscillations with the coexistence of different periods in our flux-grown $\mathrm{SmB}_{6}$ samples (for details of sample preparation and experimental methods, see Appendix A). The angle-resolved field dependencies of the magnetic torques $\tau$ are shown in Fig. 1(b). We convert the measured capacitance $C(B, \theta)$ to torque by the relation $\tau \propto 1 / C$. The absolute value of the torque signal is calibrated by a zero-field rotation in which the weight of sample $m$ generates a change in the capacitance of $\Delta(1 / C) \propto m g l \cos \phi$. Here, $l$ is the length of the cantilever beam and $\phi$ is the sample tilt angle between the magnetic field and the crystalline [001] direction, as described in Fig. 1(a).

There are several interesting features in the angledependent behavior of $\tau(B)$. First, it is apparent that the nonoscillatory background of $\tau(B)$ changes its sign abruptly at $\phi=0^{\circ}$ and $90^{\circ}$, while at $\phi=45^{\circ}$ there is another sign change, but it is much smoother. We argue that this is most (a)

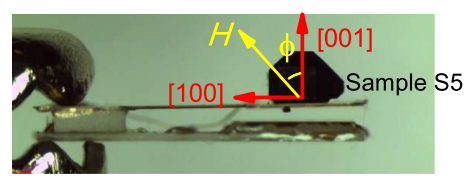

(c)

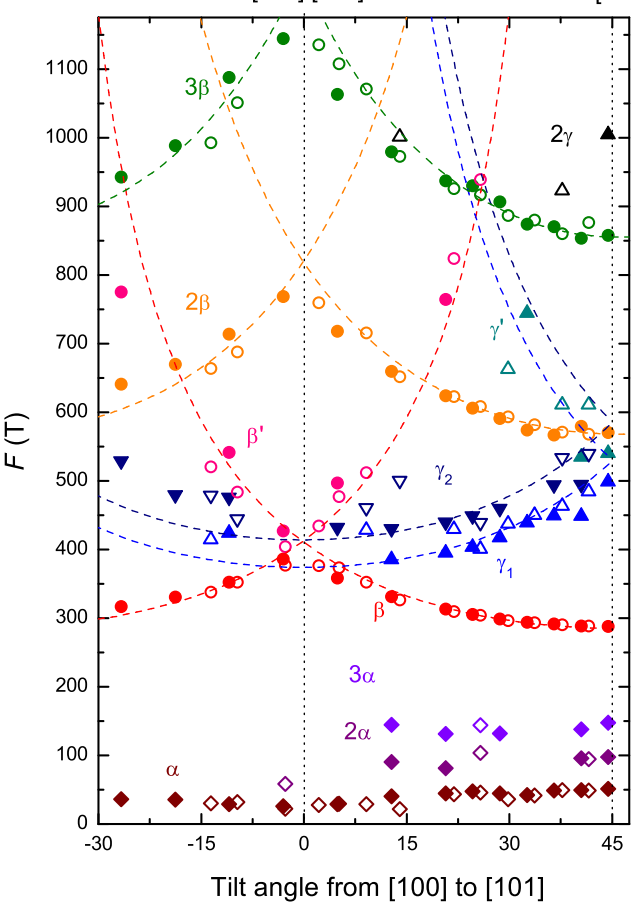

(b)

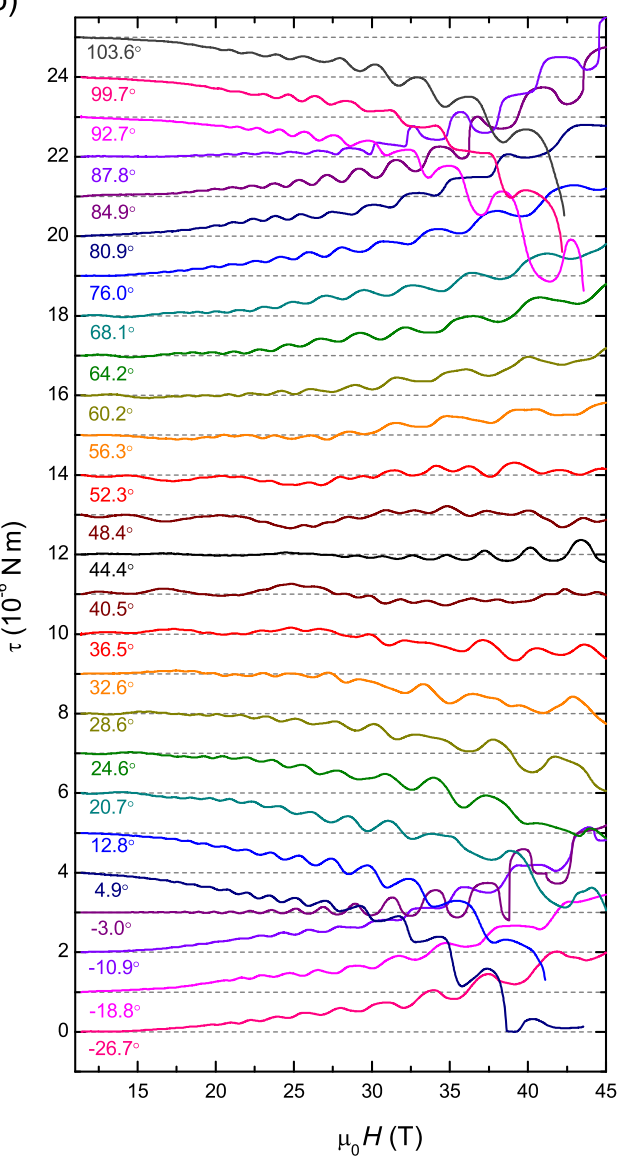

FIG. 1. Torque measurements on $\mathrm{SmB}_{6}$. (a) Photograph showing the beryllium-copper cantilever and the SmB 6 single crystal $\mathrm{S} 5$ we use for the torque magnetometry measurement. In this setup, the magnetic field is rotated in the (010) plane of the sample. Arrows sketch the definition of the tilt angle $\phi$. (b) Magnetic torque of sample S5 measured up to $45 \mathrm{~T}$ at different tilt angles. The torque curves are shifted vertically for clarity. The absolute value of torque is calibrated by the sample weight. (c) Angular dependence of all resolved dHvA oscillation frequencies on the fast Fourier transform (FFT) of $M_{\text {eff }}$ with field rotated in (010) plane. FFT peaks are indexed with the same labels as in Ref. [12]. Solid symbols denote the data points at $\phi<45^{\circ}$, while hollow symbols are data taken at $\phi>45^{\circ}$. Harmonics of branches $\alpha, \beta$, and $\gamma$ are presented by diamonds, circles, and triangles, respectively. Dashed lines are fittings based on 2D FS model: $F=F_{0} / \cos \left(\phi-\phi_{0}\right)$. For branch $\beta, \phi_{0}= \pm 45^{\circ}$ and $F_{0}=285$ T. The two "split" branches $\gamma_{1}$ (blue) and $\gamma_{2}$ (navy) both have symmetric axis along [100], i.e., $\phi_{0}=0^{\circ}$ and $90^{\circ}$, while $F_{0}$ is $374 \mathrm{~T}$ for $\gamma_{1}$ and $414 \mathrm{~T}$ for $\gamma_{2}$. 
likely due to a bulk magnetic susceptibility anisotropy between the cubic [100] and [101] directions. Second, the $\mathrm{dHvA}$ oscillations on the torque curves also change sign at $\phi=0^{\circ}, 45^{\circ}$, and $90^{\circ}$. This "flipped" dHvA pattern across certain magnetic field directions [see $\tau(B)$ curves at $\phi=$ $40.5^{\circ}$ and $48.4^{\circ}$ in Fig. 1(b) as a typical reference] hardly reflects a sudden jump on the phase of quantum oscillation, while a more natural explanation is a direction change of the oscillatory torque vector $\vec{\tau}=\vec{M} \times \vec{B}$ that happens at these angles. Both a 3D electronic system with susceptibility anisotropy along [100] and [101] and a 2D diamagnetic system can exhibit such an oscillatory torque flip at $\phi=$ $\pm(N / 4) \pi$ ( $N$ can be any integer). Third, the amplitude of dHvA oscillation is considerably large. At above $35 \mathrm{~T}$, the oscillatory torque $\Delta \tau$ is roughly $(0.5-1) \times 10^{-6} \mathrm{~N} \mathrm{~m}$, corresponding to an effective magnetic moment $\Delta M_{\text {eff }}=$ $\Delta \tau / \mu_{0} H$ of approximately $(1-2) \times 10^{-8} \mathrm{~A} \mathrm{~m}^{2}$.

Such a large dHvA oscillation amplitude basically rules out the possibility of "false quantum oscillation" coming from the $\mathrm{Al}$ flux incorporated inside the sample (Appendix B), but also gives some difficulties to the 2D surface state interpretation. In a standard analysis on 2D electron systems, the magnetization oscillation has an amplitude upper limit of $e \hbar n_{2 \mathrm{D}} / \pi m^{*}$ for a unit area. This dHvA amplitude is usually much larger than that in real materials since there are several damping factors needed to be taken into account [26]. Here, $n_{2 \mathrm{D}}$ is the 2D density of carriers that contribute to the dHvA oscillations. Using the electronic parameters calculated in our earlier work [12], a 2D magnetic moment of $\sim 1 \times 10^{-9} \mathrm{~A} \mathrm{~m}^{2}$ is estimated for a surface area of $1 \mathrm{~cm}^{2}$. Giving the millimeter size of our sample and the effective magnetic moment $\sim 1 \times 10^{-8} \mathrm{~A} \mathrm{~m}^{2}$, the discrepancy turns out to be roughly 2 orders of magnitude. Moreover, the $M_{\text {eff }}$ discussed above is only the component perpendicular to the magnetic field, $M_{\text {eff }}=M_{\perp}$, and is therefore smaller than the total magnetic moment. The puzzling large amplitude of the dHvA oscillations has been, however, reported in the confirmed 3D topological insulator $\mathrm{Bi}_{1-x} \mathrm{Sb}_{x}$ [27], though the reason that the conventional estimation based on the surface carrier density failed there is unknown [28]. Further works looking into the peculiar magnetizing properties of the topological surface state are needed to solve this question.

The fast Fourier transformation (FFT) of $M_{\text {eff }}$ show results that are consistent with our previous work [12]. Three main branches $F^{\alpha}, F^{\beta}, F^{\gamma}$ and their higher-order harmonics are resolved. The FFT peak positions are plotted in Fig. 1(c) as a function of the angle between the applied field and the crystalline equivalent [100] directions in the cubic structure of $\mathrm{SmB}_{6}$. For $F^{\beta}$ and $F^{\gamma}$, a fitting of $F=$ $F_{0} / \cos \left(\phi-\phi_{0}\right)$ can follow the behavior of $F(\phi)$ quite well, indicating a 2D nature of the related FSs. The value of $\phi_{0}$ hints that pockets $\beta$ and $\gamma$ have the symmetric axes along the equivalent [101] and [100] directions, respectively. A "split" of peak $\gamma$ is observed in a wide angle range, which may indicate a subtle magnetic breakdown (for details, see Appendix C). These results are confirmed by repeated measurements in several samples, and no sample dependence on the dHvA frequencies has ever been observed. Also, a recent tunneling spectroscopy study on $\mathrm{SmB}_{6}$ single crystals reveals two Dirac-like surface bands on the (100) surface and an additional one on the (101) surface [29], which is in agreement with our observation of three bands. The smallest orbit $\alpha$, however, has indeterminate dimension and geometry (Appendix C).

We do not resolve any dHvA frequencies higher than 2 kT (Appendix D). The high-frequency components observed in FZ-grown $\mathrm{SmB}_{6}$, which indicate large Fermi pockets with size comparable to the area of the Brillouin zone [13], are confirmed to be absent in flux-grown samples. It should be pointed out that there are still some similarities between the quantum oscillation spectra in our flux-grown samples and those in FZ-grown crystals (see Appendix $\mathrm{E}$ for details). In FZ-grown $\mathrm{SmB}_{6}$, lowfrequency oscillations were also resolved and assigned to small orbits $\rho$ and $\rho^{\prime}$ [13]. $\rho^{\prime}$ shares the same angle range with our branch $\alpha$, and $\rho$ is close to $\beta$ and $\gamma$ in our FFT spectra. As mentioned in Ref. [13], alternative possibilities are cylinderlike "neck" sections in a 3D electronic structure, or elongated ellipsoidal FSs. A similar elaborated comparison of the angle dependence of $\beta / \gamma$ and $\rho$ has been made in Ref. [17], which shows inconclusive results in distinguishing the effectiveness of the 2D and 3D model. Therefore, the angular dependence of the oscillation frequencies cannot determine the origin of quantum oscillations in $\mathrm{SmB}_{6}$. As we demonstrate later, the angular dependence of the oscillation amplitudes indicates that the oscillations most likely arise from the surface state.

The angular dependence of the FFT amplitude of the dHvA oscillation branch $F^{\beta}$ is plotted in Fig. 2(a). Here, we use a tilt angle $\theta$ with a different definition: the angle between $H$ and the [101] direction of the crystal, as depicted in the inset of Fig. 2(a). As expected for an effective magnetization extracted from magnetic torque data, this amplitude will drop to zero if the total magnetization vector is parallel or antiparallel to the applied magnetic field, which results in no "effective" component to be detected. In Fig. 2, this is shown to happen at both $H \|$ [101] and $H \perp$ [101], once again suggesting that pocket $\beta$ is related to the (101) planes. With the magnetic field rotated approximately $60^{\circ}$ away from the symmetric axis [101], the FFT amplitude is reduced by a factor of 20-100, consistent with the behavior of the $2 \mathrm{D}$ topological surface state in $\mathrm{Bi}_{1-x} \mathrm{Sb}_{x}$ [27] (a detailed comparison is provided in Appendix F). Beyond this tilt angle, the FFT can hardly pick up the oscillation signal from the corresponding Fermi pocket.

The most interesting feature of the $\theta$-dependent FFT amplitude is the absence of fourfold symmetry corresponding to the cubic crystal structure. That is, from the [100] to [001] direction $\left(\theta<-45^{\circ}\right)$ and from the [001] 

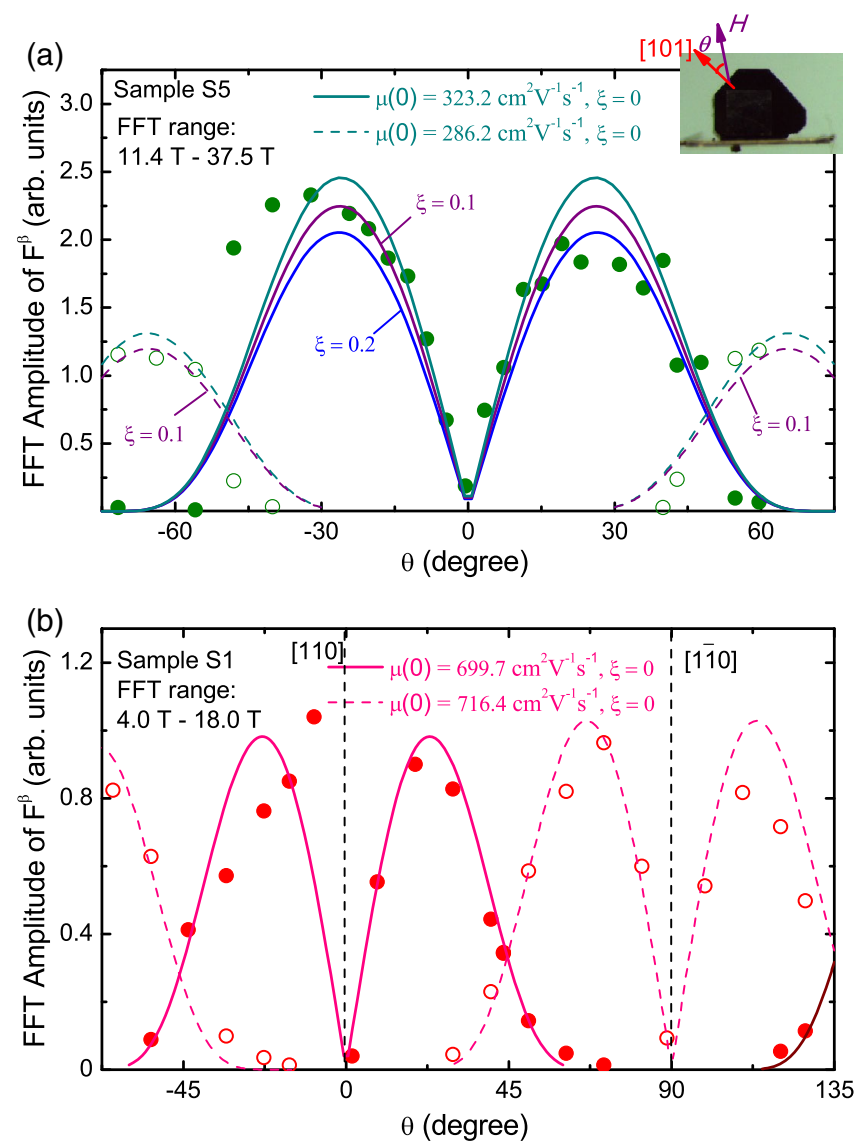

FIG. 2. Angular dependence of oscillation amplitudes. (a) The amplitude of peak $\beta$ in the FFT spectra of $M_{\text {eff }}$, plotted against the tilt angle $\theta$ between $H$ and [101] direction. Inset shows how $\theta$ is defined. The solid (fitted by the solid line) and hollow (fitted by the dashed line) symbols denote the amplitude of $F^{\beta}$ assumed to come from surfaces $(101)(\overline{1} 0 \overline{1})$ and $(10 \overline{1})(\overline{1} 01)$, respectively. The fittings (dark cyan) are made using the 2D LK model in Eq. (1) and yield $\xi=0$. Results with $\xi=0.1$ (purple) and 0.2 (blue) are also shown for comparison. (b) Amplitude analysis using the same model applied on the data of an old $\mathrm{SmB}_{6}$ sample, $\mathrm{S} 1$, measured up to 18 T. Data are extracted from Fig. S3 in Ref. [12].

to [100] direction $\left(\theta>+45^{\circ}\right)$, the amplitude of $F^{\beta}$ is obviously smaller than that between [100] to [001] $\left(-45^{\circ}<\right.$ $\theta<+45^{\circ}$ ). The broken fourfold rotational symmetry in the (010) plane strongly suggests that the oscillation frequency may not have a bulk origin. Also, this inequivalence between axes [101] and [101] shows sample dependence. In Fig. 2(b), we summarize the angle-resolved dHvA amplitudes in another $\mathrm{SmB}_{6}$ single crystal, $\mathrm{S} 1$, measured with the same experimental setup. The twofold feature in Fig. 2(b) is much weaker, suggesting that the symmetry breaking is more related to the sample instead of the cantilever magnetometry setup.

A reasonable interpretation of this symmetry breaking is the surface origin of $F^{\beta}$. In our magnetic torque measurement, the signal from two parallel surfaces [e.g., (101) and

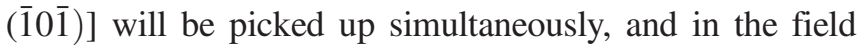

rotation in the (001) plane we can obtain the magnetic response from two individual sets of surfaces, which are perpendicular to each other. These two sets of surfaces are prone to have different plane impurity densities and subsequently different carrier scattering rates that can apparently affect the amplitude of the quantum oscillation (Appendix F). Here, we analyze the angular dependence of the dHvA amplitude of $F^{\beta}$ using a 2D LK model [26]:

$\Delta M_{\perp}(\theta) \propto \frac{\sin \theta}{\cos ^{2} \theta} \exp \left(-\frac{\pi}{\mu(0) B \cos \theta}\right) \exp (-\xi \cos \theta)$,

where $M_{\perp}$ is the effective magnetic moment picked up in torque measurement, $\mu(0)$ is the carrier mobility at $\theta=0$, $\mu(0)=e \tau_{s}(0) / m^{*}(0)$, and $\xi=\pi \lambda B / \mu(0)$. This model takes the $1 / \cos \theta$ anisotropy of the cyclotron mass $\mathrm{m}^{*}(\theta)$ as the main contribution to the angular dependence of oscillation amplitude. The comprehensive simplification process of this model, in which we consider the anisotropy of each term in the LK formula in the 2D case, is presented in Appendix F. We also apply Eq. (1) to the dHvA data of the topological surface state as well as the bulk state in $\mathrm{Bi}_{1-x} \mathrm{Sb}_{x}$ reported in Ref. [27]; the fittings we show in Figs. 7(a) and 7(b) in Appendix F provide strong evidence that Eq. (1) is a valid model in describing the two dimensionality of electronic states and can effectively track the difference in the angle-dependent quantum oscillation amplitude between 2D and 3D system.

As shown by the fittings in Fig. 2, the twofold symmetry in FFT amplitude can be well described by a difference of carrier mobility $\mu(0)$ on the two perpendicular sets of surfaces. By assuming a reduction on $\mu(0)$ of $\simeq 11.5 \%$ [Fig. 2(a)], the nearly $50 \%$ amplitude suppression is reproduced even for a 25\% larger surface area [estimated from the sample geometry in the inset of Fig. 1(a)]. This result substantially supports the 2D nature of oscillation branch $\beta$, as even an elongated ellipsoidal FS shows an evident deviation at high tilt angle (Appendix F). Furthermore, the effective fitting by using zero or a small value of $\xi$ reveals an ignorable Zeeman attenuation of the scattering rate (Appendix F).

The mobility difference is much larger between different samples, as in sample $\mathrm{S} 2, \mu(0)$ [Fig. 7(c) in Appendix F] is more than 3 times as large as in sample S5 [Fig. 2(a)]. This sample-dependent behavior is more likely due to the varied surface impurity level within samples. As we know, the scattering on the surface of $\mathrm{SmB}_{6}$ is highly related to the surface disorder [30], and the dephasing length is different by several hundred percent in different samples [10]. Also, the carrier mobility $\mu(0)$ we obtain from the fitting is only $50 \%-70 \%$ of that calculated from the Dingle plot (see Appendix G). This discrepancy can be addressed to the complicated electron scattering mechanism in $\mathrm{SmB}_{6}$, which remains an enigma to be solved by further studies. We point out that the mobilities attained by our dHvA amplitude fitting and Dingle analysis yield the same order of magnitude and are both much higher than those obtained from transport experiments $[14,31]$. 
Finally, the fitting based on the LK formula in Fig. 2 demonstrates again the validity of the Fermi liquid theory in $\mathrm{SmB}_{6}$. The mysterious sudden enhancement of quantum oscillation amplitude in $\mathrm{SmB}_{6}$ reported by Tan et al. [13] has attracted much attention as a rare 3D example of the deviation from the LK formula, which has been suggested as a reflection of unconventional quantum oscillation [19-21,32,33]. In our dHvA studies down to $40 \mathrm{mK}$, however, such behavior is not repeated, even though we apply up to $45 \mathrm{~T}$ magnetic field, stronger than that used in Ref. [13]. In Fig. 3, we summarize the temperature dependence of dHvA oscillations at $\phi=32.6^{\circ}$ : the capacitance $C(B)$ [Fig. 3(a)], the oscillatory magnetic torque $\tau_{\text {osc }}$ [inset of Fig. 3(a)], and the FFT curves of $\tau_{\text {osc }}$ [Fig. 3(b)] all show almost no discernible difference at varied temperatures between 41 and $656 \mathrm{mK}$. None of the dHvA branches we resolve have any abnormal enhancement in this temperature range. Figure 3(c) summarizes the evolution of the FFT amplitude. The light effective masses for both $F^{\beta}$ and $F^{\gamma}$ together with the low $T$ give a temperature damping factor $R_{T}$ very close to 1 . As a result, the oscillation amplitude is generally a constant with the relative change almost ignorable. We also track the evolution of the FFT amplitude of $F^{\beta}$ from $350 \mathrm{mK}$ up to $30 \mathrm{~K}$, at a tilt angle of $\phi=41^{\circ}$, as shown in Fig. 3(d). Assuming that in the 2D LK formula the damping factor related to temperature is the same as that for the 3D case [34], the overall behavior can be fitted by the LK formula with an effective mass of $0.138 \mathrm{~m}_{e}$, consistent with our former report [12]. The weak temperature dependence of dHvA amplitude below $300 \mathrm{mK}$ is confirmed by measurements in different samples and at various tilt angles, which is shown in Fig. 9 in Appendix H.

The distinct physical phenomena observed in flux-grown and FZ-grown $\mathrm{SmB}_{6}$ are quite intriguing and confusing. The FZ growth are reported to induce a small portion of $\mathrm{Sm}$ vacancies in $\mathrm{SmB}_{6}$ single crystal [35-37]. Subsequently, a slight difference on the $\mathrm{Sm}$ valence on the surface can be established, which in turn modifies the Kondo interaction near the surface [16,17]. Evidences of incomplete Kondo coupling in $\mathrm{SmB}_{6}$, especially at the vicinity of the surface, have been discovered [14,36,38,39]. Actually, in a topological Kondo insulator, both light and heavy surface states can be supposed to appear by varying the detailed band
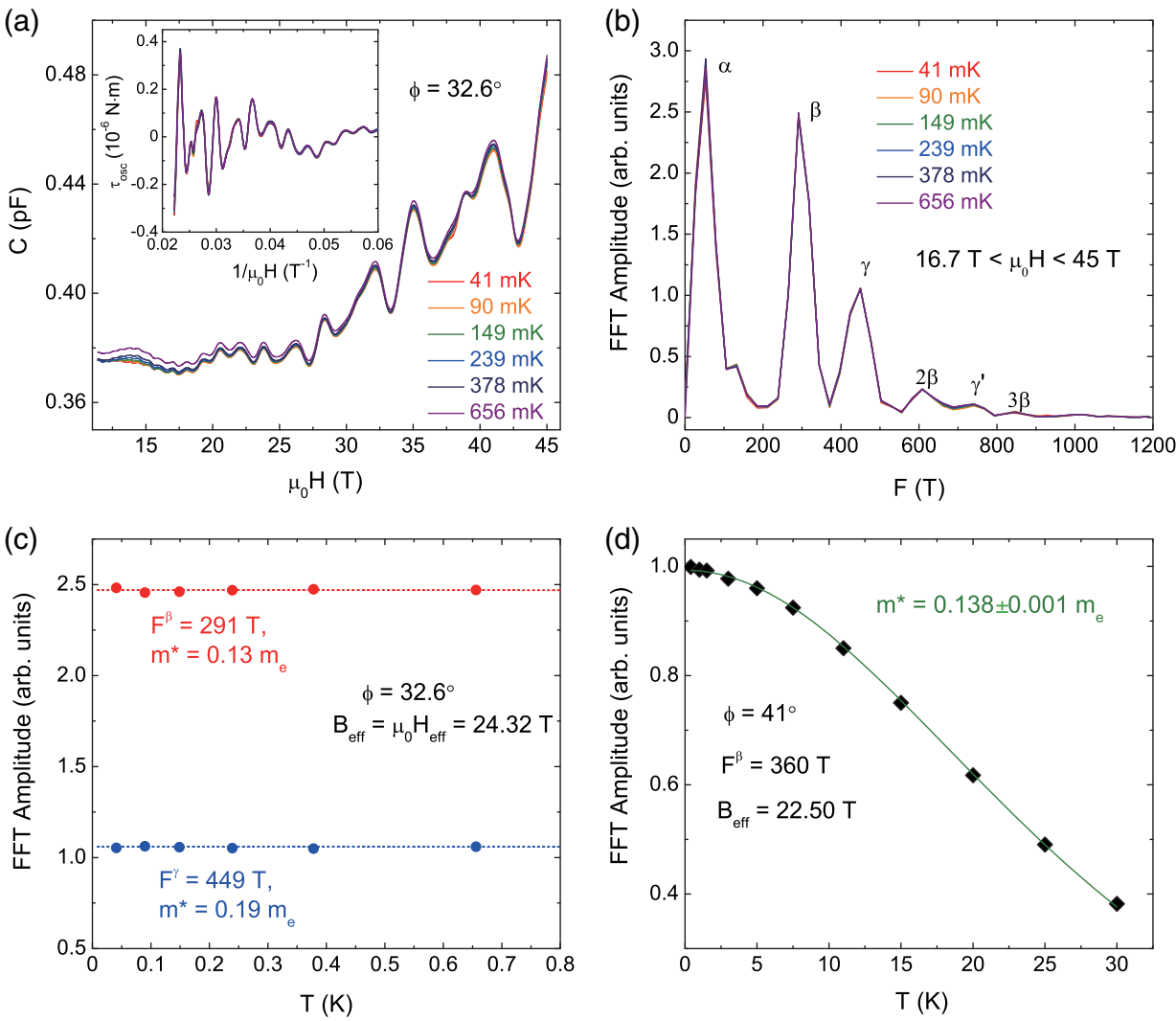

FIG. 3. Temperature dependence of oscillation amplitudes. (a) Capacitance signals in Sample S5 measured up to 45 T at different temperatures ranged from $41 \mathrm{mK}$ to $656 \mathrm{mK}$. The tilt angle for this data set is $\phi=32.6^{\circ}$. Inset: The oscillatory part of magnetic torque extracted from the capacitance curves, as a function of inverse magnetic field. A polynomial background is subtracted. (b) The FFT amplitude curves of magnetic torque shown in the inset of (a) in a field range between $16.7 \mathrm{~T}$ and $45 \mathrm{~T}$. (c) The FFT amplitudes of $F^{\beta}$ and $F^{\gamma}$ plotted as a function of temperature. Dashed lines are fittings based on Lifshitz-Kosevich formula with effective mass $m^{*}=0.13 m_{e}$ and $0.19 m_{e}$ for oscillation branches $\beta$ and $\gamma$, respectively. (d) Temperature dependence of the FFT amplitude of $F^{\beta}$ tracked up to $30 \mathrm{~K}$ at $\phi=41^{\circ}$. Fitting by LK formula yield an effective mass of $0.138 \mathrm{~m}_{e}$. 
parameters [40]. Besides this valence variation scenario induced by nonstoichiometry in the framework of Kondo physics, another interpretation of the inconsistencies between our results and those reported in FZ-grown crystals by Tan et al. [13] is attributed to different disorder levels. Recent theory points out that a topological Kondo insulator can be restored from an exotic Skyrme insulator, in which dHvA oscillation is contributed by scalar charge neutral particles, by introducing a certain degree of disorder [24]. The absence of high-frequency oscillations in our sample can also be attributed to the higher scattering rate induced by the impurity or defects. Furthermore, the deviation of LK behavior in FZ-grown samples was taken as evidence for the exotic nature of oscillations [13]; we note that a similar double-step feature was observed in topological nodal semimetal ZrSiS [41], in which the Fermi surface nesting leads to two LK behavior with two different effective masses. This is a more realistic origin than the various unconventional quantum oscillation models [19-21,23,24,32,33]. Overall, the topological surface state explanation is still the most natural one for all of our observations of the magnetic quantum oscillations in flux-grown $\mathrm{SmB}_{6}$.

In summary, magnetic torque data of the Kondo insulator $\mathrm{SmB}_{6}$ measured in an intense magnetic field at dilution refrigerator temperature has been investigated comprehensively. The amplitude of the main $\mathrm{dHvA}$ oscillation branch $\beta$, which shows $1 / \cos \phi$ angular dependence in frequency, displays a broken fourfold symmetry with field rotating in the crystalline (010) plane. The angle-dependent oscillation amplitude can be fitted by a standard 2D LK model with respect to each set of (101) planes. The carrier scattering rate obtained from the fittings is significantly different between samples as well as surfaces on the same sample. The dHvA oscillations are also fully saturated at low temperature, implying small carrier masses. Our results indicate that multiple 2D light electron states exist on the surfaces of $\mathrm{SmB}_{6}$.

\section{ACKNOWLEDGMENTS}

This work is mainly supported by the National Science Foundation under Grant No. DMR-1707620 (magnetization measurement), by the Office of Naval Research through the Young Investigator Prize under Grant No. N00014-15-1-2382 (electrical transport characterization), and the National Science Foundation Major Research Instrumentation under Grant No. DMR-1428226 (supports the equipment of the thermodynamic and electrical transport characterizations). C.S. and X.H.C. thank the National Key R\&D Program of the MOST of China (Grant No. 2016YFA0300201). The development of the torque magnetometry technique in intense magnetic fields was supported by the Department of Energy under Award No. DE-SC0008110. Some experiments were performed at the National High Magnetic Field Laboratory, which is supported by NSF Cooperative Agreement No. DMR084173, by the State of Florida, and by the DOE. The experiment in NHMFL is funded in part by a QuantEmX grant from ICAM and the Gordon and Betty Moore Foundation through Grant No. GBMF5305 to Z.X., T. A., C. T., L.C., and L.L. We are grateful for the assistance of Tim Murphy, Glover Jones, Hongwoo Baek, and Ju-Hyun Park of NHMFL. T. A. thanks the Nakajima Foundation for support. B. L. acknowledges support by the National Science Foundation Graduate Research Fellowship under Grant No. F031543.

\section{APPENDIX A: MATERIALS AND METHODS}

$\mathrm{SmB}_{6}$ single crystals are grown by the $\mathrm{Al}$ flux method [25]. The chunks of Sm (99.95\%), the powder of boron (99.99\%), and $\mathrm{Al}(99.99 \%)$ are mixed together with a mass ratio of $1: 6: 400$, and then loaded into an alumina crucible. The entire mixture is heated to $1550^{\circ} \mathrm{C}$ and then stays at this temperature for 2 days before cooling down to $600{ }^{\circ} \mathrm{C}$ at $5{ }^{\circ} \mathrm{C}$ per hour. During all the preparing and heating progress, the mixture is kept in the argon gas. After being cooled to room temperature, the samples with $\mathrm{Al}$ flux are soaked in the dense $\mathrm{NaOH}$ solution to remove the $\mathrm{Al}$ flux, and then washed by dilute $\mathrm{HNO}_{3}$ solution. The samples are characterized by $\mathrm{x}$-ray diffraction to determine the orientation. Upon cooling from room temperature to ${ }^{3} \mathrm{He}$ temperature, the resistivity of our $\mathrm{SmB}_{6}$ samples is enhanced by more than 4 orders of magnitude, and a resistive plateau shows up below $3.5 \mathrm{~K}$ [15]. Data discussed in this work are mainly taken from $\mathrm{SmB}_{6}$ sample S5, which has a size of $2.1 \times 1.6 \times 1.2 \mathrm{~mm}^{3}$ and hosts large (100) and (101) surfaces. A smaller sample labeled as S6 is also measured, but the results show no significant differences and the signal quality is lower due to the smaller quantum oscillation amplitudes. All the samples we use are as-grown single crystals without cleaving or polishing.

The high magnetic field torque magnetometry measurements are carried out using the capacitance method in the National High Magnetic Field Laboratory (NHMFL), Tallahassee. The $\mathrm{SmB}_{6}$ samples are glued to a beryllium-copper cantilever with a thickness of $0.025 \mathrm{~mm}$. The variation in the capacitance between the cantilever and a fixed gold film reflects the bending of the cantilever, from which the magnetic torque can be obtained. The setup with sample S5 attached is shown in Fig. 1(a). Such devices are put into a rotator and then loaded into a dilution fridge in a hybrid magnet which can apply magnetic field up to $45 \mathrm{~T}$. The cantilevers are rotated with magnetic field in the crystalline (010) plane.

We measure the capacitance change with sweeping magnetic field via two methods. The frequently used AndeenHagerling AH2700A digital capacitance bridge usually has a noise level of $10^{-4} \mathrm{pF}$ in our experimental environment, and the automatic balancing is slow, which means it may not be suitable to pick up the weak high-frequency dHvA oscillations in $\mathrm{SmB}_{6}$. As an alternative, we chose the General Radio analog capacitance bridge combined with the Stanford Research SR124 analog lock-in amplifier. By balancing the 
starting capacitance $C_{0}$ manually and reading the voltage change during field sweeping, we can achieve a better resolution with the noise level reduced by 1 order of magnitude. Also, this allows for a continuous reading of the cantilever response.

\section{APPENDIX B: EXCLUSION OF THE FLUX-INDUCED QUANTUM OSCILLATION}

Extrinsic quantum oscillations introduced by $\mathrm{Al}$ flux trapped inside the sample has been reported in $\mathrm{CaB}_{6}$ [42]. The existence of epitaxially oriented $\mathrm{Al}$ flakes in the fluxgrown $\mathrm{SmB}_{6}$ single crystals has also been confirmed, with a percentage of $2-4$ wt $\%$ [37]. However, the dHvA amplitude of single-crystalline aluminum is known to be $\Delta \tau \simeq$ $3.5 \times 10^{-7} \mathrm{~N} \mathrm{~m}$ at $4.2 \mathrm{~K}$ under $B=2 \mathrm{~T}$, for the strongest oscillation branch $\gamma_{5}$ in a sample with the mass of $45 \mathrm{mg}$ [43]. Considering the reported effective mass $m^{*} / m_{e}=0.18$ and Dingle temperature $T_{D}=0.8 \mathrm{~K}$ for this band in aluminum, there is an amplifying factor of approximately $60 \times$ in torque for the condition of $B=40 \mathrm{~T}$ and $T=45 \mathrm{mK}$. It means the weight of incorporated $\mathrm{Al}$, if it contributes to all the dHvA signals shown in Fig. 1(b), should be $\sim 2 \mathrm{mg}$. Since the weight of $\mathrm{SmB}_{6}$ sample $\mathrm{S} 5$ is $12.9 \mathrm{mg}$, the amount of cocrystallized Al could be as large as $15 \mathrm{wt} \%(58.4 \mathrm{~mol} \%)$, which is much larger than that revealed by an $\mathrm{x}$-ray diffraction study [37]. Consequently, the dHvA patterns in Fig. 1(b) are more likely to be intrinsic.

There is extra evidence against the flux-induced extrinsic quantum oscillations. First, there is no two-peak feature in any of the $\gamma$ branches in the dHvA oscillations of aluminum $[43,44]$, whereas such a peak split has been observed in our flux-grown $\mathrm{SmB}_{6}$ samples [Fig. 4(a)]. Second, there are evident discrepancies between the angular dependence of oscillation frequencies in $\mathrm{SmB}_{6}$ and $\mathrm{Al}$, as reported before [12]. Also, the resemblance of $\mathrm{dHvA}$ frequencies in fluxgrown samples and floating-zone-grown samples (which are grown free of $\mathrm{Al}$; see Appendix E) supports that the oscillations are intrinsic.

\section{APPENDIX C: SPLITTING ON $\gamma$ AND THE LOW-FREQUENCY OSCILLATION $\alpha$}

There are two adjacent FFT peaks at the location of branch $\gamma$, as shown in Fig. 4(a), with a frequency interval of $43 \pm 5 \mathrm{~T}$ for most of the angles. We label the peak with lower frequency $\gamma_{1}$ and the high-frequency one $\gamma_{2}$. Such a phenomenon is also presented in our old data, though under lower magnetic field (up to $18 \mathrm{~T}$ ) the splitting is less clear [12]. The inset of Fig. 4(a) gives a comparison of the FFTs with different end points, and the splitting appears to be unambiguous only for a cutoff field higher than $30 \mathrm{~T}$. The origin of this observation is unclear. If the FS $\gamma$ is a quasitwo-dimensional one and has a small periodic warping along the $k_{z}$ direction, two extrema of the FS cross-sectional area can result in two quantum oscillation frequencies that
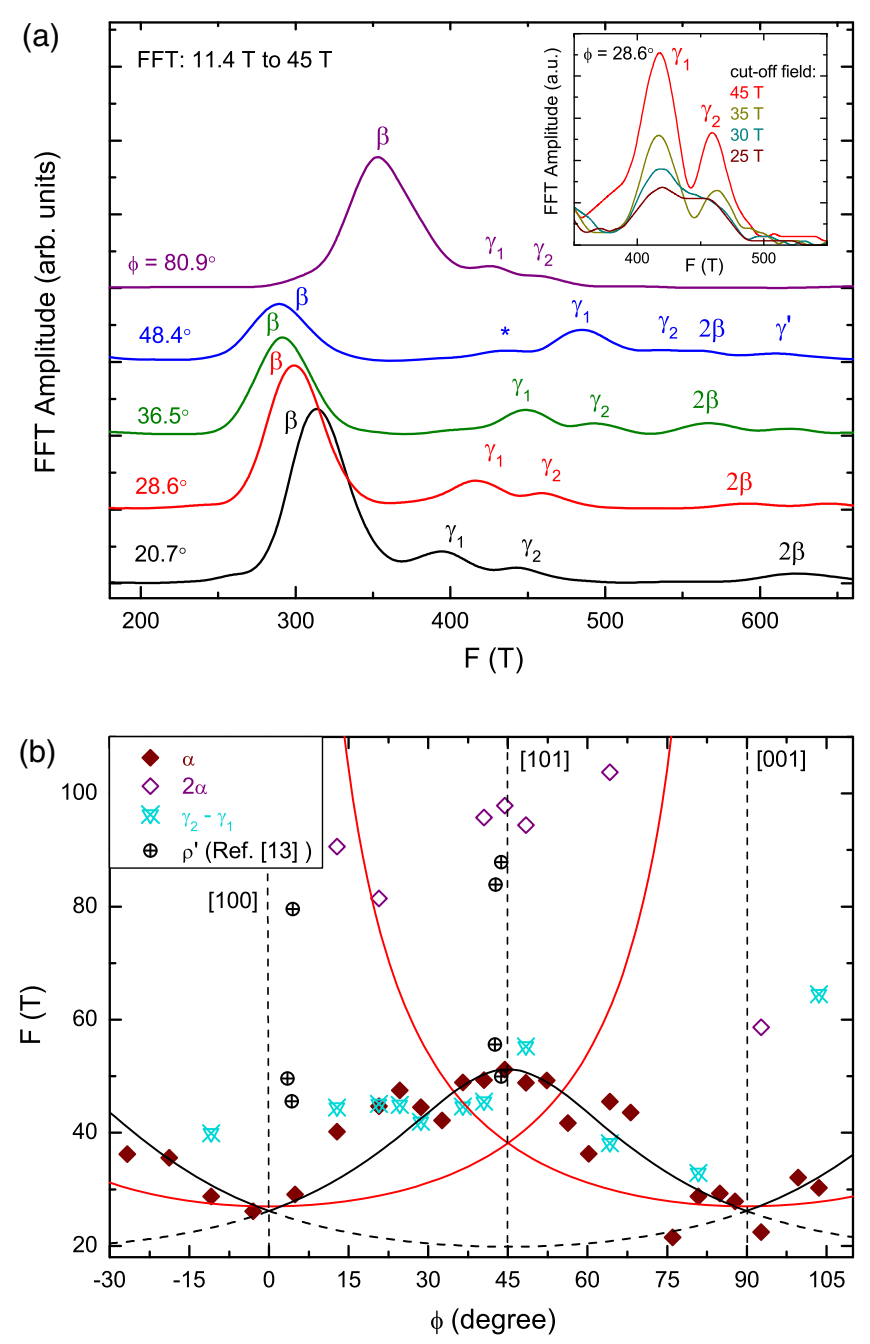

FIG. 4. (a) The FFT spectra of $M_{\text {eff }}$ for various tilt angles. Two peaks on the high-frequency side of the major peak $\beta$ are labeled as $\gamma_{1}$ and $\gamma_{2}$, respectively. One unknown small feature on the $\phi=$ $48.4^{\circ}$ at $435 \mathrm{~T}$ is marked by a star. Inset: The FFT spectrum at $\phi=28.6^{\circ}$, between $11.4 \mathrm{~T}$ and a varied cutoff field. The split of peak $\gamma$ is more evident as the cutoff field becomes larger. (b) The angle dependence of lowest frequency oscillation branch $\alpha$ and its second harmonic, plotted together with the frequency interval between split $\gamma_{1}$ and $\gamma_{2}$ and data of frequency $\rho^{\prime}$ extracted from Ref. [13]. The red curves are fittings using a 2D Fermi surface (FS) model, whereas the black curves are 3D fittings based on an ellipsoid FS. For the 3D model, we follow the previous report in which the geometry of the FSs contributing to $\rho^{\prime}$ are figured out to be small ellipsoidal pockets with their long axes along the cubic [101] directions [13]. The fitting (black solid line) gives a ratio of long axis (along [101] ) to short axis (along [101]) of 2.58. However, the other set of equivalent FSs with long axis along [101] (black dashed line) are absent from our data.

are close to each other [45,46]. In this scenario, however, the two extreme areas have different curvature in the angle dependence and can cross together at certain angles. These expectations are absent in our data [see the triangle symbols in Fig. 1(c)]. We suggest a more plausible explanation that the two peaks $\gamma_{1}$ and $\gamma_{2}$, respectively, come from two sets of 
electron orbits on (100) surfaces. Since the pockets $\alpha$ and $\gamma$ have identical symmetric axes along [100], one possibility is that the split is originated in a magnetic breakdown between them, i.e., $\gamma_{2}=\gamma_{1}+\alpha$.

We notice that the interval between the two split $\gamma$ branches shows insignificant change with varying field directions [Fig. 1(c)]. As exhibited in Fig. 4(b), the small angle dependence of $\gamma_{2}-\gamma_{1}$ almost coincides with that of $F^{\alpha}$, which raises the possibility of magnetic breakdown between orbits $\gamma_{1}$ and $\alpha$. Taking into account that the splitting of $\gamma_{2}$ is apparent only under high magnetic field [inset of Fig. 4(a)], magnetic breakdown is a rather promising explanation. With the orbit area differed by 1 order of magnitude, however, magnetic breakdown is unlikely to happen if $\gamma$ and $\alpha$ are centered at the same momentum position in the Brillouin zone. It is probable that one of the two orbits is located away from the high-symmetry points in momentum space, but in this case additional frequencies like $\gamma_{1}+n \alpha$ or $\alpha+n \gamma_{1}$ are supposed to be observed according to the crystal symmetry. Actually, they are absent in our FFT spectra. At this stage, the reason for the splitting and the locations of orbits $\gamma$ and $\alpha$ remain unclear.

The smallest orbit $\alpha$ resolved from the FFT of the torque curves has an indeterminate geometry. In Fig. 4(b), we try to simulate the angle dependence of $F^{\alpha}$ by both the 2D cylinder model and 3D elongated ellipsoid model. The 2D FS model cannot exactly follow the frequency increase from the [100] direction to the [101] direction, whereas 3D fittings based on an ellipsoidal FS with the long axis along the crystalline [101] direction can track the data fairly well. Considering the cubic symmetry of the crystal structure, equivalent FSs with the long axis along [101] should exist as well. However, they are totally absent in our FFT analysis. Additionally, the FFT peaks with higher frequencies (hollow symbols in Fig. 4), which are identified as the second harmonics here, show up only in the vicinity of the [101] direction where the oscillation amplitude of $\alpha$ is the strongest. This behavior suggests those peaks in the range of 80-110 T are harmonics in nature instead of the diverging $F^{\alpha}$ from one of the same set of FSs with the symmetry axis $90^{\circ}$ away [as $\beta^{\prime}$ and $\gamma^{\prime}$ plotted
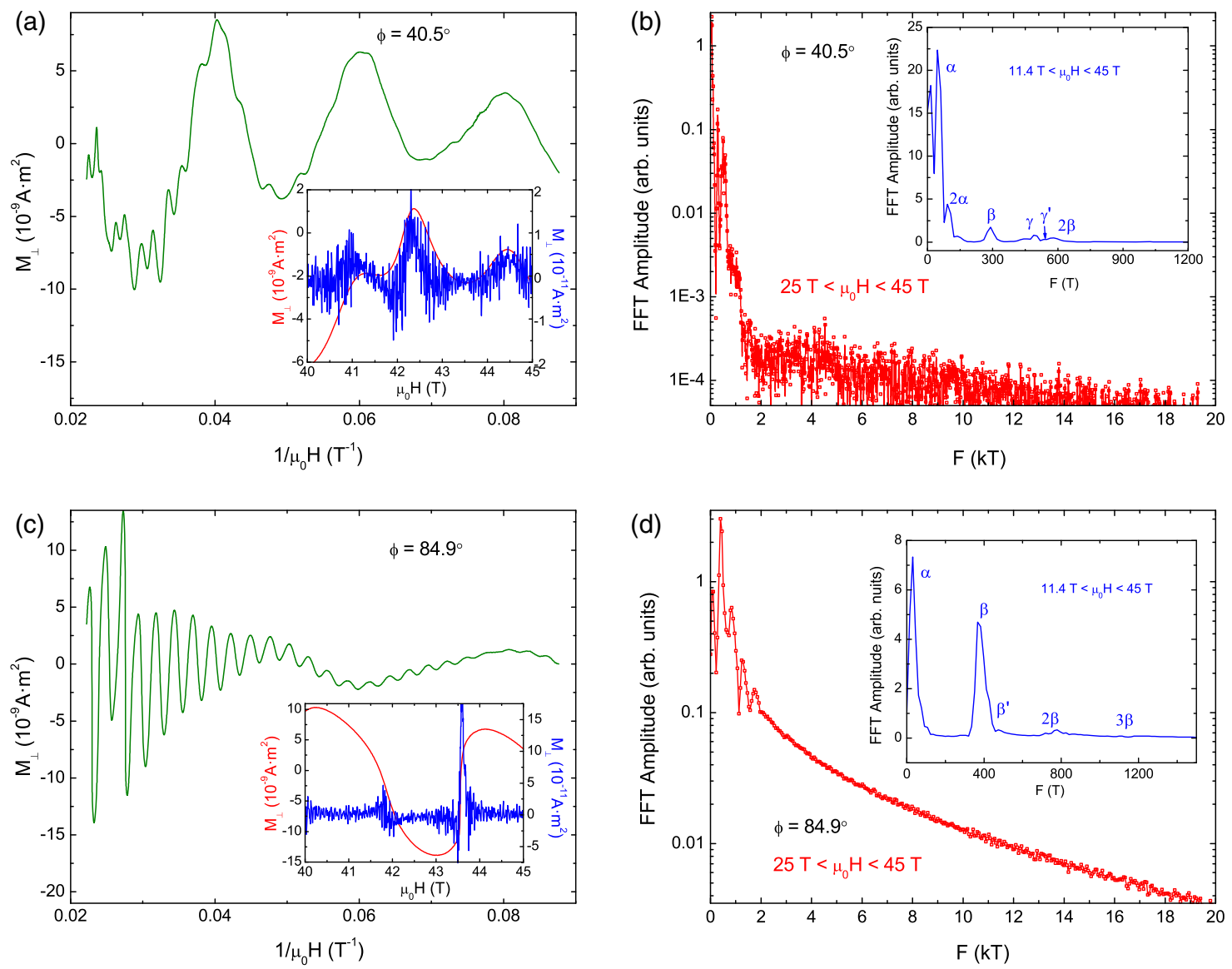

FIG. 5. Absence of high-frequency oscillations. The oscillatory effective magnetization $M_{\text {eff }}$ as a function of inverse magnetic field at (a) $\phi=40.5^{\circ}$ and (c) $\phi=84.9^{\circ}$. Polynomial backgrounds are subtracted from the raw data. Insets: Low-frequency component of $M_{\text {eff }}$ obtained by a low-pass FFT filter with threshold frequency $2 \mathrm{kT}$ (red) and the residual component after subtracting the low-frequency oscillation from $M_{\text {eff }}$ (blue), both shown in a magnetic field range between 40 and $45 \mathrm{~T}$. The FFT results of the oscillatory part of $M_{\text {eff }}$ at (b) $\phi=40.5^{\circ}$ and (d) $\phi=84.9^{\circ}$ between 25 and $45 \mathrm{~T}$. No oscillation frequency higher than $2 \mathrm{kT}$ can be resolved from the background at both tilt angles. Insets: Low-frequency peaks with $F<1200 \mathrm{~T}$ in a full range FFT from 11.4 to $45 \mathrm{~T}$. 
in Fig. 1(c) as well as in Ref. [12] ]. We also add the data reported in Ref. [13] to Fig. 4(b), though considering the different sample rotation direction in the two studies, we select only the angles close to $0^{\circ}$ ([100]) and $45^{\circ}$ ([101]). According to Tan et al., the smallest orbit $\rho^{\prime}$ is assigned to a small ellipsoid inside the "neck" connecting the large FSs [13]. However, judging from the consistency in Fig. 4(b), it is arguable that the $\rho^{\prime}$ branch is corresponding to our $\alpha$ and its second harmonic.

\section{APPENDIX D: ABSENCE OF HIGH-FREQUENCY dHvA OSCILLATIONS}

Oscillation branches with frequencies higher than $2 \mathrm{kT}$, which have been detected in the floating-zone furnace-grown $\mathrm{SmB}_{6}$ single crystals [13], are completely missing in our measurement. Actually, the frequencies between 1 and $2 \mathrm{kT}$ are already rather weak in our FFT spectra. In Fig. 5, we show the analysis for two field orientations, i.e., $\phi=40.5^{\circ}$, at which the low-frequency branch $\alpha$ is strong but $\beta$ and $\gamma$ are relatively weak, and $\phi=84.9^{\circ}$, where branch $\beta$ has a large spectral weight. At both angles the capacitance is measured by an analog capacitance bridge. The oscillatory part of $M_{\perp}$, the perpendicular component of magnetization, is dominated by the "slow" dHvA oscillations [Figs. 5(a) and 5(c)]. After subtracting those "slow" components (with $F<1200 \mathrm{~T}$ ), the residual magnetization term is barely noise with the amplitude approximately $10^{-11} \mathrm{~A} \mathrm{~m}^{2}$, that is, $0.1 \%-0.2 \%$ of the total oscillatory $M_{\perp}$ [see the inset of Figs. 5(a) and 5(c)]. No periodic small wiggles can be isolated that can indicate the existence of fast quantum oscillations. On the FFT of $M_{\perp}$, everything with frequency higher than $2 \mathrm{kT}$ sinks into the background noise that is roughly $1 / 1000$ of the main peak height and no features can be resolved, even when we take the FFT in a high field range [Figs. 5(b) and 5(d)].

\section{APPENDIX E: COMPARISON BETWEEN THE dHvA FREQUENCIES IN FLUX-GROWN AND FLOATING-ZONE-GROWN SAMPLES}

In Fig. 6, we provide a comparison of the results reported by two groups on the dHvA frequencies resolved in $\mathrm{SmB}_{6}$ single-crystal samples grown via different approaches, i.e., Al-flux crystals studied by Li et al. [12] and FZ crystals studied by Tan et al. [13]. Measurements are taken with the same technique and experimental conditions $[12,13]$. As is confirmed by Fig. 5, the high-frequency components with $F>2 \mathrm{kT}$ are not detected in the flux-grown samples. On the other hand, the low-frequency FFT peaks in these two data sets are generally comparable, with data points falling into the same frequency range, and the overall trend of angular dependence also shows some similarities. It highly suggests

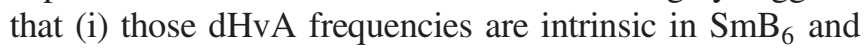
(ii) the two works are looking into the quantum oscillations from the same electronic states.

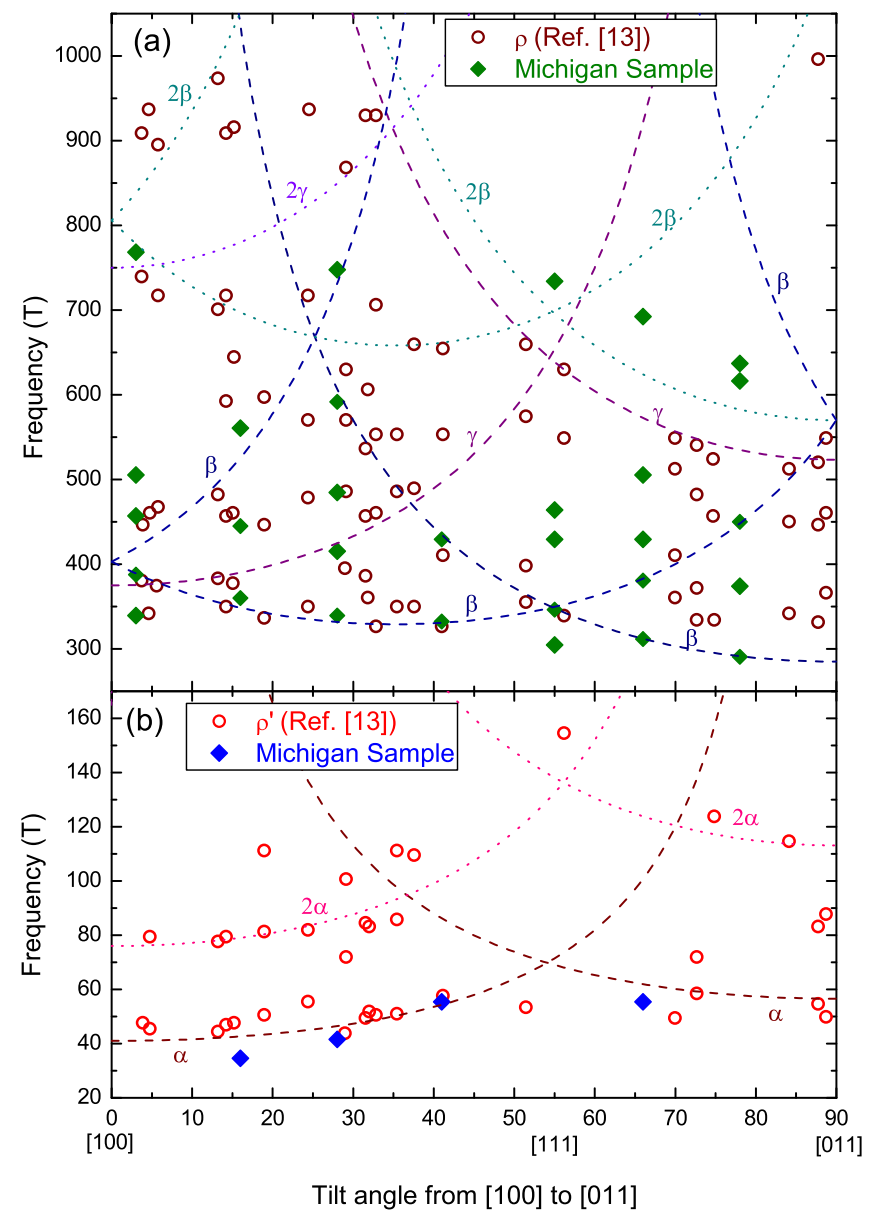

FIG. 6. A comparison of the FFT peaks resolved from the magnetic torque data in the floating-zone-grown $\mathrm{SmB}_{6}$ single crystals, extracted from Ref. [13], and the Al-flux-grown samples studied in Ref. [12]. In (a) and (b) the FFT peaks $\rho$ and $\rho^{\prime}$ in Ref. [13] are plotted, respectively, together with the dHvA oscillation features in the flux-grown samples that are within the same frequency range. In both data sets, the magnetic field is rotated from crystal [100] axis towards [011] axis. Dashed lines are fittings by the 2D cylinder FS model [12], and dotted lines are fittings of the harmonics.

\section{APPENDIX F: ANGLE DEPENDENCE OF dHvA OSCILLATION AMPLITUDE IN 2D ELECTRON SYSTEM}

For a 2D electron system, given the condition of $F / B \gg 1$, the amplitude of longitudinal magnetization quantum oscillations can be approximately described by a 2D LK expression [34,47-49]:

$M_{\|}=-A \sum_{p=1}^{\infty}\left(\frac{1}{2 \pi p}\right) R_{T} R_{D} R_{S} \sin \left[2 \pi p\left(\frac{F}{B}-\gamma\right)\right]$,

where $R_{T}=X_{p} / \sinh \left(X_{p}\right), X_{p}=2 \pi^{2} p k_{B} m^{*} T / e \hbar B, R_{D}=$ $\exp \left(-2 \pi^{2} k_{B} m^{*} T_{D} / e \hbar B\right), T_{D}=\hbar / 2 \pi k_{B} \tau_{Q}$ is the Dingle temperature, $A$ is a parameter proportional to quantum 
oscillation frequency $F$, and $R_{S}=\cos \left(p \pi g m^{*} / 2 m_{e}\right)$ is the spin-splitting factor. Here, $m_{e}$ is the free-electron mass and $\tau_{Q}$ the quantum oscillation relaxation time. In this work, we study the magnetic torque, and the effective magnetization extracted from the torque signal is $M_{\text {eff }}=M_{\perp}$. According to Ref. [26],

$$
M_{\perp}=-\frac{1}{F} \frac{\partial F}{\partial \theta} M_{\|}
$$

which serves as the starting point of our $\mathrm{dHvA}$ oscillation amplitude analysis.

There are three independent parameters in Eq. (F2) that are functions of the magnetic field tilt angle $\theta: F=F(\theta)$, $m^{*}=m^{*}(\theta)$, and $\tau_{Q}=\tau_{Q}(\theta)$. The dHvA frequency $F$ appears in the universal coefficient of all the harmonics, $(1 / F)(\partial F / \partial \theta) A$, in which $A \propto F$. According to our fitting in Fig. 2(b), $F^{\beta}(\theta)$ shows the typical characterization of a 2D FS, i.e., $F^{\beta}(\theta) \propto 1 / \cos \theta$. Therefore, we have

$$
\frac{1}{F} \frac{\partial F}{\partial \theta} A \propto \frac{\partial F}{\partial \theta} \propto \frac{\sin \theta}{\cos ^{2} \theta} .
$$

The effective mass $m^{*}$ is included in all three amplitude factors of $R_{T}, R_{D}$, and $R_{S}$. For a parabolic-band system, the definition of effective mass is $m^{*}=\left(\partial^{2} E / \partial k^{2}\right)^{-1}$, whereas, for a linear dispersive $2 \mathrm{D}$ electron system such as the surface state of a topological insulator, we can use the expression as follows $[28,50]$ :

$$
m^{*}=\frac{\hbar^{2}}{2 \pi}\left(\frac{\partial S(E)}{\partial E}\right)_{E=E_{F}},
$$

where $S(E)$ is the cross-sectional area of the 2D FS perpendicular to the field vector. Note that $S(E) \propto F$ varies as $1 / \cos \theta$, but the energy dispersion and carrier density will not change with the sample rotation in magnetic field, assuming the band dispersion relation is invariant along all directions and there is no magnetic-field-induced modification in any of the bands. It means for 2D electron systems we have $m^{*} \propto 1 / \cos \theta$, which is equally effective for the conventional parabolic and the Dirac-like band dispersion $[27,51]$. We check the anisotropy of effective mass in two $\mathrm{SmB}_{6}$ single crystals (the FFT plots of these two samples can be found in Figs. S3-S5 in Ref. [12]), and the results for pocket $\beta$ are $m^{*} / m_{e}=0.124(0.122)$ at $\theta=14.6^{\circ}$ and $m^{*} / m_{e}=0.140(0.147)$ at $\theta=34.8^{\circ}$ for sample S1 (S2). The relative offset of $m^{*}(\theta)$ in regards to the expected $1 / \cos \theta$ behavior is therefore $1.9 \%$ and $4.2 \%$ for samples $\mathrm{S} 1$ and S2, respectively. Considering the sample misalignment and the LK fitting error, this result is quite reasonable, and we can also give an estimation of the effective mass at $\theta=0^{\circ}: m_{0}=m^{*}(0) \simeq 0.120 m_{e}$.

To simplify the model, we take the Dingle factor $R_{D}$ as the only amplitude factor that is effectively influenced by the angle-dependent $m^{*}(\theta)$. This approximation is actually sensible for the following reasons. Giving the small value of $m_{0}$ for pocket $\beta$ and the low environment temperature, the angular dependence of $R_{T}$ is almost negligible. For $m_{0} / m_{e}=0.12$ and $T=40 \mathrm{mK}$, the value of $R_{T}$ is very close to 1 with an offset smaller than $0.1 \%$ except for the angle range $\left|\theta-90^{\circ}\right|<1^{\circ}$. In our measurement, the oscillation signal from the $\beta$ branch on one surface cannot be detected with the field $\theta>75^{\circ}$ away from the normal direction of the relating surface, as shown in Fig. 2. Hence, the factor $R_{T}$ can be safely treated as a constant in our fitting. We also leave out the spin-splitting factor $R_{S}$ since we do not have a reliable estimation of the Landé factor $g$ for the light Fermi pocket $\beta$. If this pocket is a topological surface state, this factor will hardly play any role in affecting the quantum oscillation amplitude, because $R_{S}$ comes from the superposition of oscillations from the split Landau levels (i.e., spin-up and spin-down) [26]. In a topological surface state there is no spin degeneracy at $k \neq 0$; accordingly, the Zeeman effect shifts the position of the Landau levels instead of causing the splitting $[27,28,52]$, which consequently results in no reduction on the amplitude of oscillation.

The angular dependence of the relaxation time $\tau_{Q}(\theta)$ is more complicated. We separate the magnetic field into two components, the in-plane field $H_{\|}=H \sin \theta$ and the outof-plane field $H_{\perp}=H \cos \theta$. The first term is known to have no significant transport response from the topological surface state in the absence of the hybridization between the top and bottom surfaces [53], which can be completely neglected in our bulk single crystals. Theoretically, the inplane magnetic field will only shift the position of the surface Dirac point in momentum space $[54,55]$ and cause a net in-plane spin polarization [56]. A deformation of the Fermi pocket corresponding to the spin-density redistribution is also suggested [57]. In all, the spin momentum locking and the prohibition of backscattering in topological surface states is not destroyed in an in-plane magnetic field. The case is totally different for the second term, the out-ofplane component, which can break the time-reversal symmetry and lift the protection of the topological nontriviality. In this case, the backscattering is reintroduced, and the electron-impurity scattering is enhanced by the Zeeman-energy-related spin canting [58]. The transport scattering rate takes the form

$$
\frac{1}{\tau_{\mathrm{tr}}}=\frac{1}{\tau_{0}}\left(1+\lambda B^{2} \cos ^{2} \theta\right)
$$

where $\lambda$ is a system parameter related to the $g$ factor and Fermi energy.

Taking into account all the angle-dependent parameters we discuss above, we can give a fitting model of the quantum oscillation amplitude of $M_{\perp}$ for the fundamental harmonic: 


$$
\begin{aligned}
\Delta M_{\perp}(\theta) & =\frac{1}{F(\theta)} \frac{\partial F(\theta)}{\partial \theta}\left(\frac{A(\theta)}{2 \pi}\right) R_{D}(\theta) \\
& \propto \frac{\sin \theta}{\cos ^{2} \theta} \exp \left(-2 \pi^{2} k_{B} m^{*} T_{D} / e \hbar B\right) \\
& =\frac{\sin \theta}{\cos ^{2} \theta} \exp \left(-\frac{\pi}{\mu(0) B \cos \theta}\right) \exp (-\xi \cos \theta),
\end{aligned}
$$

where $\mu(0)$ is the carrier mobility at $\theta=0: \mu(0)=$ $e \tau_{s}(0) / m^{*}(0)$, and $\xi=\pi \lambda B / \mu(0)$. This expression is the same as Eq. (1). One needs to mention that the transport relaxation time $\tau_{\text {tr }}$ in Eq. (F5) is different from the quantum oscillation relaxation time $\tau_{Q}$ we use in the Dingle factor, as the former one is more sensitive to backscattering [59]. Also, the field dependence of $\tau_{\text {tr }}$ in Eq. (F5) is basically a weak-field approximation. As a simplification, in the fitting model Eq. (F6) we assume that $\tau_{\text {tr }}$ and $\tau_{Q}$ share the same field dependence in the magnetic field range in our measurement. The detailed field effect on the scattering in topological surface states still needs further investigation.

To examine the validity of our model, we apply it to the quantum oscillation amplitudes in a well-known topological insulator $\mathrm{Bi}_{1-x} \mathrm{Sb}_{x}$, reported by Taskin and Ando, in which both $2 \mathrm{D}$ and $3 \mathrm{D}$ FSs can be resolved in dHvA measurement [27]. Since the data in Ref. [27] were taken by SQUID magnetometer, the oscillations were detected on longitudinal magnetization; consequently, the fitting model Eq. (F6) should be modified to

$$
\Delta M_{\|}(\theta) \propto \frac{1}{\cos \theta} \exp \left(-\frac{\pi}{\mu(0) B \cos \theta}\right) .
$$

Here, we drop the field-dependent mobility term due to lack of information. Relying on the electronic parameters presented in Ref. [27], the carrier mobilities of the 2D surface state $\left[F_{1}\right.$ in Fig. $7($ a) $]$ and $3 \mathrm{D}$ bulk state $\left[F_{2}\right.$ in Fig. $\left.7(\mathrm{~b})\right]$ are 5.5 and $1.2 \mathrm{~m}^{2} \mathrm{~V}^{-1} \mathrm{~s}^{-1}$, respectively. Taking these mobilities as fitting parameters in Eq. (F7), the curve in Fig. 7(a) can roughly track the fast decrease of dHvA amplitude when field is rotated towards the bisectrix plane down to $\theta \simeq 20^{\circ}$. However, in Fig. 7(b), the attenuation of the dHvA amplitude is obviously much slower than that expected in our $2 \mathrm{D}$ model below $\theta<50^{\circ}$. It should be mentioned that the frequency $F_{2}$ comes from a highly anisotropic ellipsoidal FS in $\mathrm{Bi}_{1-x} \mathrm{Sb}_{x}$ : the length of its longest semiaxis (along crystal axis $C_{3}$ ) is 8.5 and 17.7 times of the other two semiaxes, respectively [27]. The fittings in Fig. 7 indicate that even for such an extremely elongated FS, our model can effectively distinguish it from a real 2D cylinder FS.

The 2D LK formula we use in data fitting is theoretically a low magnetic field approximation. In a 2D system, if the number of electrons is kept constant, the chemical potential is prone to be pinned in the highest occupied Landau level and also oscillates with increasing field [26]. This chemical potential oscillation will cause strong deviation from LK (a)

(b)

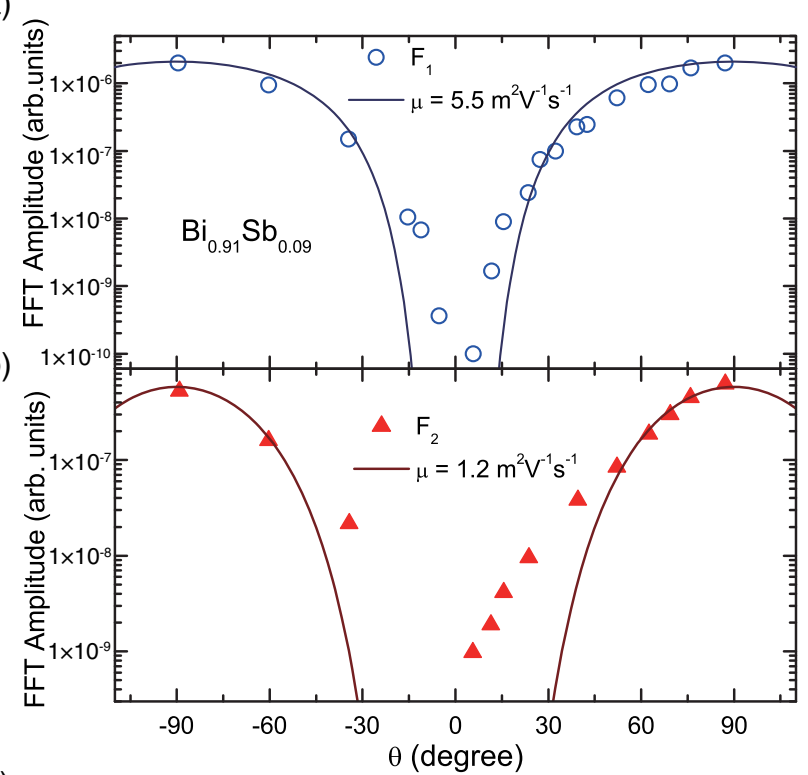

(c)

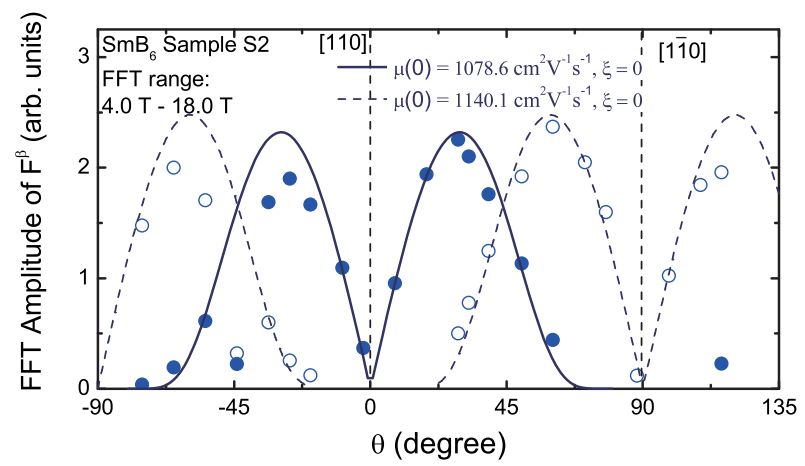

FIG. 7. The dHvA oscillation amplitudes of (a) 2D surface state on the bisectrix plane and (b) $3 \mathrm{D}$ ellipsoidal bulk FS in $\mathrm{Bi}_{1-x} \mathrm{Sb}_{x}$, fitted by Eq. (F7). Data points are extracted from Ref. [27]. Tilt angle $\theta$ is the angle between the magnetic field, which is rotated in the binary plane, and the crystal axis $C_{3}$. The carrier mobility in each panel is calculated from the parameters obtained in the same work. (c) Fitting of the angle-dependent amplitude of FFT peak $\beta$ in $\mathrm{SmB}_{6}$ sample $\mathrm{S} 2$ by Eq. (F6), with parameter $\xi=0$. Definitions of $\mu_{0}$ and $\xi$ are the same as in Fig. 2. Data are extracted from Fig. S4 in Ref. [12].

theory at high field, in which the position of chemical potential is assumed to be fixed [47,60]. Such deviation has been found in quasi-2D organic compounds [34,47,61] as well as in the cuprate high-temperature superconductor $\mathrm{YBa}_{2} \mathrm{Cu}_{3} \mathrm{O}_{6+x}$ [62]. In topological insulators, however, this behavior has not ever been reported or discussed. An analytical analogue of the LK formula, with well-defined $R_{T}$ and $R_{D}$, has been established for the 2D Dirac-like electron system [63], and is effectively used in graphene [50]. Conventional LK analysis is widely applied and accepted in the study of quantum oscillations in topological insulators $[27,28,64,65]$. As for our data, the upper limit of magnetic field $B(45 \mathrm{~T})$ is much lower than the oscillation frequencies $F^{\beta}$ and $F^{\gamma}$; therefore, the low-field condition $\Delta E_{n} \ll E_{F}$ is 
fulfilled for these two bands (here, $\Delta E_{n}$ is the energy interval between Landau levels and $E_{F}$ the Fermi energy). In addition, the sharp sawtoothlike oscillation patterns expected for clean 2D systems are missing in our measurements, and the LK description works well in fitting the temperature dependence of the oscillation amplitude [12]. Given the reasonable modeling of the angular dependence of the oscillation amplitude in the surface state of $\mathrm{Bi}_{1-x} \mathrm{Sb}_{x}$, we conclude that the LK model in our analysis is a correct model.

For the value of $B$ in the fittings, we still use the averaged inverse field [66] as the effective value: $B_{\text {av }}=17.48 \mathrm{~T}$ in Fig. 2(a) and $B_{\text {av }}=6.54 \mathrm{~T}$ in Figs. 2(b) and 7(c). For all three samples, the fittings are reasonably good, though not perfect, with the parameter $\xi=0$. We also make curves with finite values of $\xi$ and other fitting parameters unchanged in Fig. 2(a). It appears that an acceptable value is $\xi \lesssim 0.1$, corresponding to $\lambda \lesssim 5.89 \times 10^{-5}$, and the enhancement on $1 / \tau$ is less than $\simeq 12 \%$ at $45 \mathrm{~T}$. The small Zeeman effect in the scattering rate is qualitatively consistent with the calculation for Bi-based 3D topological insulators [58]. We note that with an appreciable Zeeman effect there will be visible splitting of the peaks or valleys in the dHvA oscillation patterns. Early work also suggested that a large Zeeman effect makes the Landau level indexing plot nonlinear [67]. None of these effects are observed in our results of the quantum oscillation patterns in $\mathrm{SmB}_{6}$ [12]. Nonetheless, the small mismatch in the fittings in Fig. 2 can be assigned to the subtle effects that are ignored in our model, such as the Zeeman term, as well as the sample misalignment in the measurement.

The effective fittings by a 2D LK model [Eq. (F6)] are an essential evidence against the bulk origin of $F^{\beta}$. As mentioned above, our model describes a fast amplitude damping with field rotating away from the symmetric axis of FS. The elongated 3D FS in $\mathrm{Bi}_{1-x} \mathrm{Sb}_{x}$ with FS cross-sectional areas 8.5 times different between two perpendicular directions shows apparent deviation from the fitting curves [Fig. 7(b)]. The supposed 3D orbit $\rho$ in $\mathrm{FZ} \mathrm{SmB}_{6}$ samples [13], which shares the same frequency range with our $\beta$ branch, has a cross-sectional area difference of $\sim 3.3$ between [101] and $[10 \overline{1}]$ directions. Such a moderate anisotropy cannot give the fitting results shown in Figs. 2 and 7(c).

\section{APPENDIX G: DINGLE PLOT AND SCATTERING RATE DIFFERENCE BETWEEN SAMPLES}

Figure 8 gives a brief summary of the Dingle temperature $T_{D}$ in the three samples $\mathrm{S} 5, \mathrm{~S} 1$, and $\mathrm{S} 2$, derived from the slope of $\ln \left(\Delta M_{\perp} / R_{T}\right)$ versus $1 / B$. The linearity of the Dingle plot suggests that the field modification on $\tau_{Q}$ is almost ignorable. While the quantum oscillation mobility $\mu=e \hbar / 2 \pi k_{B} m^{*} T_{D}$ is $50 \%-100 \%$ larger than the fitting parameter $\mu(0)$ in Fig. 2, the relative magnitude of mobility within the three samples is the same for the two approaches. The discrepancies between mobilities attained by

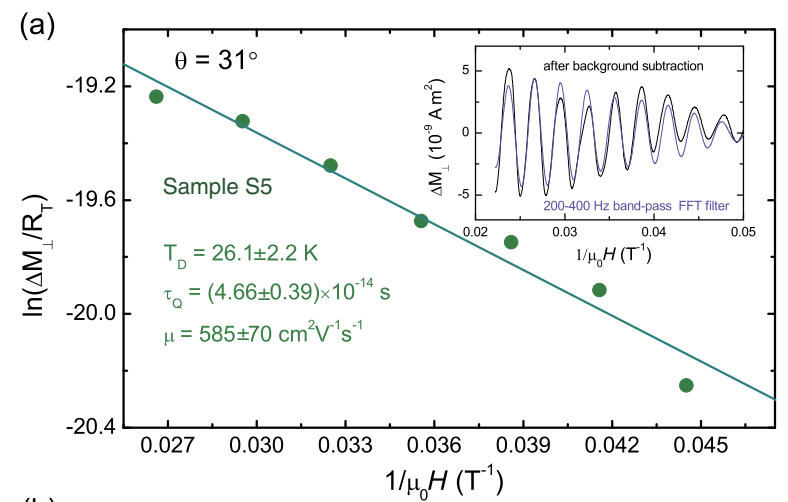

(b)

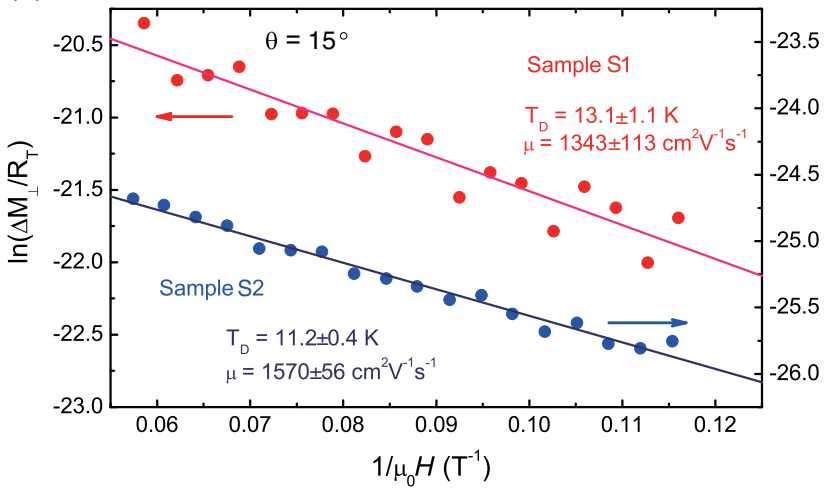

FIG. 8. Dingle plots. (a) Dingle plot $\ln \left(\Delta M_{\perp} / R_{T}\right)$ vs $1 / B$ for sample S5 at $\theta=31^{\circ}$. Solid line is the linear fit which has a slope of $-\left(2 \pi^{2} k_{B} m^{*} T_{D}\right) / e \hbar$. The carrier mobility $\mu$ is calculated as $\mu=e \tau_{Q} / m^{*}=e \hbar / 2 \pi k_{B} m^{*} T_{D}$. Inset: Raw data of $M_{\perp}$ after subtracting the nonoscillatory background and the bandpassfiltered oscillation pattern used in the Dingle plot. (b) Dingle plots for sample $\mathrm{S} 1$ and $\mathrm{S} 2$ at $\theta=15^{\circ}$.

different experimental methods is a famous conundrum in $\mathrm{SmB}_{6}$. In transport measurement, much lower mobilities have been reported, which vary from several tens of $\mathrm{cm}^{2} \mathrm{~V}^{-1} \mathrm{~s}^{-1}$ [68] to $120-140 \mathrm{~cm}^{2} \mathrm{~V}^{-1} \mathrm{~s}^{-1}$ [14,31], and no quantum oscillation has ever been observed. These confusing phenomena may suggest a complicated scattering mechanism in this material. Nonetheless, our magnetic quantum oscillation experiment has clearly proved that light carriers with relative high mobilities reside in $\mathrm{SmB}_{6}$, most likely on the surfaces.

The difference of $\mathrm{dHvA}$ amplitudes among samples is appreciably large. While sample S5 shows $\Delta M_{\text {eff }}$ with a magnitude of $10^{-8} \mathrm{~A} \mathrm{~m}^{2}$ [Fig. 1(b)], signals from other samples can be 1-2 orders of magnitude smaller with the surface area within the same order of magnitude [12]. It is also apparent that the signal strength is not proportional to the related surface area on one sample [Fig. 2(a)]. Apart from the most likely reason of surface impurity effect on the carrier mobility, we are also aware of the complex surface reconstruction in $\mathrm{SmB}_{6}$ [69-71]. The multiple surface phases are possible to give different contributions to quantum oscillation. This information is not included in the fittings in Fig. 2. 

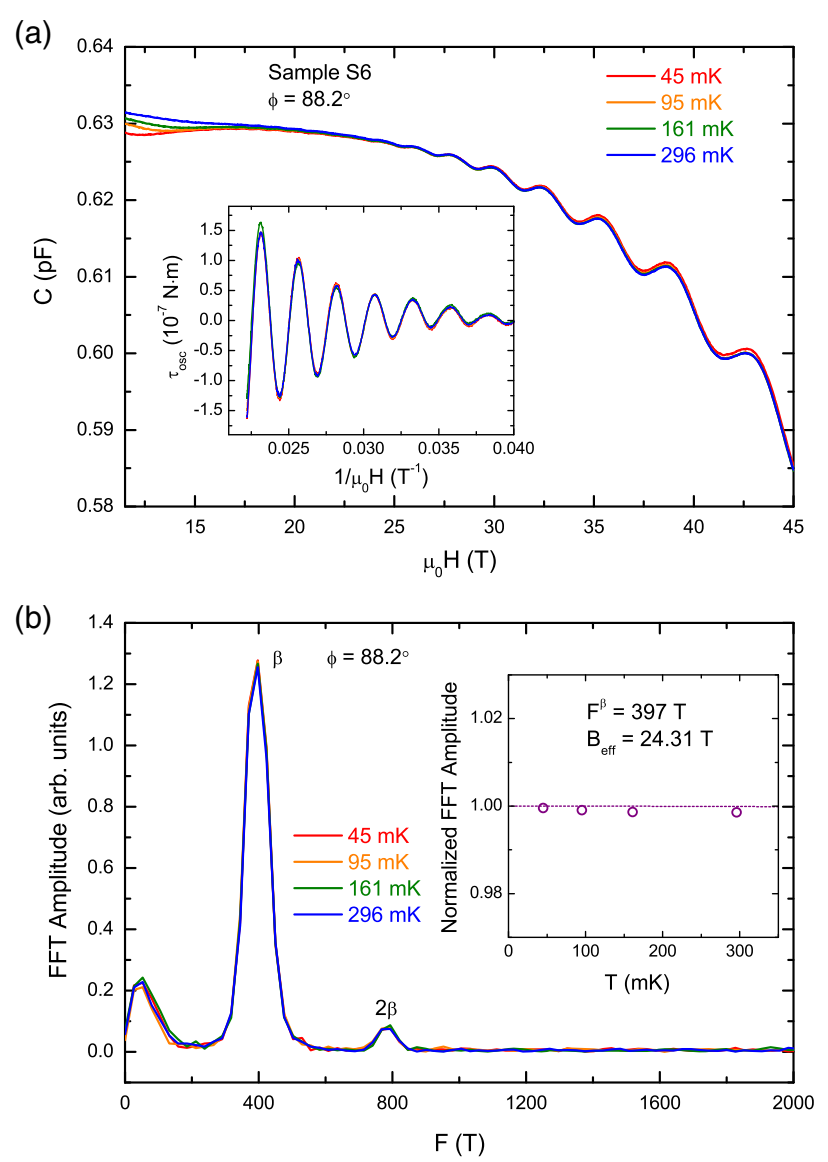

FIG. 9. (a) Magnetic torque below ${ }^{3} \mathrm{He}$ temperature in sample S6 measured by the cantilever capacitor at $\phi=88.2^{\circ}$. Inset: Oscillatory part of magnetic torque plotted against inverse magnetic field. (b) FFT curves of magnetic torque in a field range between 16.7 and 45 T. Inset: Normalized FFT amplitudes of $F^{\beta}$ as a function of temperature. Dashed line is fitting based on LK formula with effective mass $m^{*}=0.14 m_{e}$.

\section{APPENDIX H: ADDITIONAL INFORMATION OF THE TEMPERATURE DEPENDENCE OF dHvA AMPLITUDES}

The temperature dependence of the $\mathrm{dHvA}$ oscillations in $\mathrm{SmB}_{6}$ is investigated repeatedly in different samples and different magnetic field orientations. In all the measurements the dHvA amplitudes show almost temperature-independent behavior between the base temperature of the dilution fridge $(40-45 \mathrm{mK})$ and $300 \mathrm{mK}$. No considerable low-temperature $\mathrm{dHvA}$ amplitude increase [13] has been observed. Figure 9 shows the result taken at $\phi=88.2^{\circ}$, i.e., field close to the [100] direction, in sample S6. Similar to the observation shown in Fig. 3, the oscillatory magnetic torque curves at all temperatures overlap with each other. The dominating frequency at this tilt angle is $F^{\beta}=397 \mathrm{~T}$, as shown in Fig. 9(b). The amplitude attenuation of this peak is within $0.2 \%$ from 45 to $296 \mathrm{mK}$, in contrast with the previous observation of
$>80 \%$ reported by Tan et al. [13] in floating-zone-grown crystals. At this stage we confirm that such a steep increase in dHvA amplitude does not exist in our fluxgrown samples.

[1] P. S. Riseborough, Heavy Fermion Semiconductors, Adv. Phys. 49, 257 (2000).

[2] M. Dzero, K. Sun, V. Galitski, and P. Coleman, Topological Kondo Insulators, Phys. Rev. Lett. 104, 106408 (2010).

[3] M. Dzero, K. Sun, P. Coleman, and V. Galitski, Theory of Topological Kondo Insulators, Phys. Rev. B 85, 045130 (2012).

[4] A. Menth, E. Buehler, and T. Geballe, Magnetic and Semiconducting Properties of $\mathrm{SmB}_{6}$, Phys. Rev. Lett. 22, 295 (1969).

[5] T. Takimoto, $\mathrm{SmB}_{6}$ : A Promising Candidate for a Topological Insulator, J. Phys. Soc. Jpn. 80, 123710 (2011).

[6] V. Alexandrov, M. Dzero, and P. Coleman, Cubic Topological Kondo Insulators, Phys. Rev. Lett. 111, 226403 (2013).

[7] F. Lu, J. Z. Zhao, H. Weng, Z. Fang, and X. Dai, Correlated Topological Insulators with Mixed Valence, Phys. Rev. Lett. 110, 096401 (2013).

[8] M. Dzero, J. Xia, V. Galitski, and P. Coleman, Topological Kondo Insulators, Annu. Rev. Condens. Matter Phys. 7, 249 (2016).

[9] D.-J. Kim, J. Xia, and Z. Fisk, Topological Surface State in the Kondo Insulator Samarium Hexaboride, Nat. Mater. 13, 466 (2014).

[10] S. Thomas, D. J. Kim, S. B. Chung, T. Grant, Z. Fisk, and J. Xia, Weak Antilocalization and Linear Magnetoresistance in the Surface State of $\mathrm{SmB}_{6}$, Phys. Rev. B 94, 205114 (2016).

[11] N. Xu et al., Direct Observation of the Spin Texture in $\mathrm{SmB}_{6}$ as Evidence of the Topological Kondo Insulator, Nat. Commun. 5, 4566 (2014).

[12] G. Li, Z. Xiang, F. Yu, T. Asaba, B. Lawson, P. Cai, C. Tinsman, A. Berkley, S. Wolgast, Y. S. Eo, Dae-Jeong Kim, C. Kurdak, J. W. Allen, K. Sun, X. H. Chen, Y. Y. Wang, Z. Fisk, and L. Li, Two-Dimensional Fermi Surfaces in Kondo Insulator $\mathrm{SmB}_{6}$, Science 346, 1208 (2014).

[13] B. Tan, Y.-T. Hsu, B. Zeng, M. C. Hatnean, N. Harrison, Z. Zhu, M. Hartstein, M. Kiourlappou, A. Srivastava, M. D. Johannes, T. P. Murphy, J.-H. Park, L. Balicas, G. G. Lonzarich, G. Balakrishnan, and S. E. Sebastian, Unconventional Fermi Surface in an Insulating State, Science 349, 287 (2015).

[14] S. Wolgast, Y. S. Eo, T. Oztürk, G. Li, Z. Xiang, C. Tinsman, T. Asaba, B. Lawson, F. Yu, J. W. Allen, K. Sun, L. Li, Ç. Kurdak, D.-J. Kim, and Z. Fisk, Magnetotransport Measurements of the Surface States of Samarium Hexaboride Using Corbino Structures, Phys. Rev. B 92, 115110 (2015).

[15] F. Chen, C. Shang, Z. Jin, D. Zhao, Y. P. Wu, Z. J. Xiang, Z. C. Xia, A. F. Wang, X. G. Luo, T. Wu, and X. H. Chen, Magnetoresistance Evidence of a Surface State and a FieldDependent Insulating State in the Kondo Insulator $\mathrm{SmB}_{6}$, Phys. Rev. B 91, 205133 (2015). 
[16] V. Alexandrov, P. Coleman, and O. Erten, Kondo Breakdown in Topological Kondo Insulators, Phys. Rev. Lett. 114, 177202 (2015).

[17] O. Erten, P. Ghaemi, and P. Coleman, Kondo Breakdown and Quantum Oscillations in $\mathrm{SmB}_{6}$, Phys. Rev. Lett. 116, 046403 (2016).

[18] R. Peters, T. Yoshida, H. Sakakibara, and N. Kawakami, Coexistence of Light and Heavy Surface States in a Topological Multiband Kondo Insulator, Phys. Rev. B 93, 235159 (2016).

[19] J. Knolle and N. R. Cooper, Quantum Oscillations without a Fermi Surface and the Anomalous de Haas-van Alphen Effect, Phys. Rev. Lett. 115, 146401 (2015).

[20] L. Zhang, X.-Y. Song, and F. Wang, Quantum Oscillation in Narrow-Gap Topological Insulators, Phys. Rev. Lett. 116, 046404 (2016).

[21] H. K. Pal, F. Piéchon, J.-N. Fuchs, M. Goerbig, and G. Montambaux, Chemical Potential Asymmetry and Quantum Oscillations in Insulators, Phys. Rev. B 94, 125140 (2016).

[22] J. Pixley, R. Yu, S. Paschen, and Q. Si, Global Phase Diagram and Momentum Distribution of Single-Particle Excitations in Kondo Insulators, arXiv:1509.02907.

[23] G. Baskaran, Majorana Fermi Sea in Insulating $\mathrm{SmB}_{6}: A$ Proposal and a Theory of Quantum Oscillations in Kondo Insulators, arXiv:1507.03477.

[24] O. Erten, P.-Y. Chang, P. Coleman, and A. M. Tsvelik, Skyrme Insulators: Insulators at the Brink of Superconductivity, Phys. Rev. Lett. 119, 057603 (2017).

[25] P. C. Canfield and Z. Fisk, Growth of Single Crystals from Metallic Fluxes, Philos. Mag. B 65, 1117 (1992).

[26] D. Shoenberg, Magnetic Oscillations in Metals (Cambridge University Press, Cambridge, England, 2009).

[27] A. A. Taskin and Y. Ando, Quantum Oscillations in a Topological Insulator $\mathrm{Bi}_{1-x} \mathrm{Sb}_{x}$, Phys. Rev. B 80, 085303 (2009).

[28] Y. Ando, Topological Insulator Materials, J. Phys. Soc. Jpn. 82, 102001 (2013).

[29] W. K. Park, L. Sun, A. Noddings, D.-J. Kim, Z. Fisk, and L. H. Greene, Topological Surface States Interacting with Bulk Excitations in the Kondo Insulator $\mathrm{SmB}_{6}$ Revealed via Planar Tunneling Spectroscopy, Proc. Natl. Acad. Sci. U.S.A. 113, 6599 (2016).

[30] M. Dzero, M. G. Vavilov, K. Kechedzhi, and V. M. Galitski, Nonuniversal Weak Antilocalization Effect in Cubic Topological Kondo Insulators, Phys. Rev. B 92, 165415 (2015).

[31] P. Syers, D. Kim, M. S. Fuhrer, and J. Paglione, Tuning Bulk and Surface Conduction in the Proposed Topological Kondo Insulator $\mathrm{SmB}_{6}$, Phys. Rev. Lett. 114, 096601 (2015).

[32] J. Knolle and N. R. Cooper, Excitons in Topological Kondo Insulators: Theory of Thermodynamic and Transport Anomalies in $\mathrm{SmB}_{6}$, Phys. Rev. Lett. 118, 096604 (2017).

[33] H. K. Pal, Quantum Oscillations from Inside the Fermi Sea, Phys. Rev. B 95, 085111 (2017).

[34] J. Singleton, Studies of Quasi-Two-Dimensional Organic Conductors Based on BEDT-TTF Using High Magnetic Fields, Rep. Prog. Phys. 63, 1111 (2000).

[35] W. A. Phelan, S. M. Koohpayeh, P. Cottingham, J. W. Freeland, J. C. Leiner, C. L. Broholm, and T. M. McQueen, Correlation between Bulk Thermodynamic Measurements and the Low-Temperature-Resistance Plateau in $\mathrm{SmB}_{6}$, Phys. Rev. X 4, 031012 (2014).

[36] M. E. Valentine, S. Koohpayeh, W. A. Phelan, T. M. McQueen, P. F. S. Rosa, Z. Fisk, and N. Drichko, Breakdown of the Kondo Insulating State in $\mathrm{SmB}_{6}$ by Introducing Sm Vacancies, Phys. Rev. B 94, 075102 (2016).

[37] W. Phelan, S. Koohpayeh, P. Cottingham, J. Tutmaher, J. Leiner, M. Lumsden, C. Lavelle, X. Wang, C. Hoffmann, M. Siegler, N. Haldolaarachchige, D. P. Young, and T. M. McQueen, On the Chemistry and Physical Properties of Flux and Floating Zone Grown $\mathrm{SmB}_{6}$ Single Crystals, Sci. Rep. 6, 20860 (2016).

[38] Y. Nakajima, P. Syers, X. Wang, R. Wang, and J. Paglione, One-Dimensional Edge State Transport in a Topological Kondo Insulator, Nat. Phys. 12, 213 (2016).

[39] P. K. Biswas, M. Legner, G. Balakrishnan, M. C. Hatnean, M. R. Lees, D. M. Paul, E. Pomjakushina, T. Prokscha, A. Suter, T. Neupert, and Z. Salman, Suppression of Magnetic Excitations Near the Surface of the Topological Kondo Insulator $\mathrm{SmB}_{6}$, Phys. Rev. B 95, 020410(R) (2017).

[40] X.-Y. Feng, H. Zhong, J. Dai, and Q. Si, Dirac-Kondo Semimetals and Topological Kondo Insulators in the Dilute Carrier Limit, arXiv:1605.02380.

[41] S. Pezzini, M. R. van Delft, L. Schoop, B. Lotsch, A. Carrington, M. I. Katsnelson, N.E. Hussey, and S. Wiedmann, Unconventional Mass Enhancement around the Dirac Nodal Loop in ZrSiS, arXiv:1701.09119.

[42] T. Terashima, C. Terakura, Y. Umeda, N. Kimura, H. Aoki, and S. Kunii, Ferromagnetism vs Paramagnetism and False Quantum Oscillations in Lanthanum-Doped $\mathrm{CaB}_{6}$, J. Phys. Soc. Jpn. 69, 2423 (2000).

[43] C. Larson and W. Gordon, Low-Field de Haas-van Alphen Study of the Fermi Surface of Aluminum, Phys. Rev. 156, 703 (1967).

[44] N. Ashcroft, The Fermi Surface of Aluminium, Philos. Mag. 8, 2055 (1963).

[45] K. Yamaji, On the Angle Dependence of the Magnetoresistance in Quasi-Two-Dimensional Organic Superconductors, J. Phys. Soc. Jpn. 58, 1520 (1989).

[46] A. I. Coldea, J. D. Fletcher, A. Carrington, J. G. Analytis, A. F. Bangura, J.-H. Chu, A. S. Erickson, I. R. Fisher, N. E. Hussey, and R. D. McDonald, Fermi Surface of Superconducting LaFePO Determined from Quantum Oscillations, Phys. Rev. Lett. 101, 216402 (2008).

[47] N. Harrison, R. Bogaerts, P. H. P. Reinders, J. Singleton, S. J. Blundell, and F. Herlach, Numerical Model of Quantum Oscillations in Quasi-Two-Dimensional Organic Metals in High Magnetic Fields, Phys. Rev. B 54, 9977 (1996).

[48] N. Harrison, A. House, I. Deckers, J. Caulfield, J. Singleton, F. Herlach, W. Hayes, M. Kurmoo, and P. Day, de Haas-van Alphen Study of the Charge-Transfer Salt $\alpha-(\mathrm{BEDT}-\mathrm{TTF})_{2} \mathrm{KHg}(\mathrm{SCN})_{4}$ Pulsed Magnetic Fields of Up to 54 T, Phys. Rev. B 52, 5584 (1995).

[49] B. Ramshaw, B. Vignolle, J. Day, R. Liang, W. Hardy, C. Proust, and D. Bonn, Angle Dependence of Quantum Oscillations in $\mathrm{YBa}_{2} \mathrm{Cu}_{3} \mathrm{O}_{6.59}$ Shows Free-Spin Behaviour of Quasiparticles, Nat. Phys. 7, 234 (2011).

[50] K. S. Novoselov, A. K. Geim, S. Morozov, D. Jiang, M. Katsnelson, I. Grigorieva, S. Dubonos, and A. Firsov, 
Two-Dimensional Gas of Massless Dirac Fermions in Graphene, Nature (London) 438, 197 (2005).

[51] A. A. Taskin, K. Segawa, and Y. Ando, Oscillatory Angular Dependence of the Magnetoresistance in a Topological Insulator $\mathrm{Bi}_{1-x} \mathrm{Sb}_{x}$, Phys. Rev. B 82, 121302 (2010).

[52] Y.-S. Fu, T. Hanaguri, K. Igarashi, M. Kawamura, M. Bahramy, and T. Sasagawa, Observation of Zeeman Effect in Topological Surface State with Distinct Material Dependence, Nat. Commun. 7, 10829 (2016).

[53] C. J. Lin, X. Y. He, J. Liao, X. X. Wang, V. Sacksteder IV, W. M. Yang, T. Guan, Q. M. Zhang, L. Gu, G. Y. Zhang, C. G. Zeng, X. Dai, K. H. Wu, and Y. Q. Li, Parallel Field Magnetoresistance in Topological Insulator Thin Films, Phys. Rev. B 88, 041307 (2013).

[54] I. Garate and M. Franz, Magnetoelectric Response of the Time-Reversal Invariant Helical Metal, Phys. Rev. B 81, 172408 (2010).

[55] A. A. Zyuzin, M. D. Hook, and A. A. Burkov, Parallel Magnetic Field Driven Quantum Phase Transition in a Thin Topological Insulator Film, Phys. Rev. B 83, 245428 (2011).

[56] A. Sulaev, M. Zeng, S.-Q. Shen, S. K. Cho, W. G. Zhu, Y. P. Feng, S. V. Eremeev, Y. Kawazoe, L. Shen, and L. Wang, Electrically Tunable In-Plane Anisotropic Magnetoresistance in Topological Insulator BiSbTeSe 2 Nanodevices, Nano Lett. 15, 2061 (2015).

[57] L.-X. Wang, Y. Yan, L. Zhang, Z.-M. Liao, H.-C. Wu, and D.-P. Yu, Zeeman Effect on Surface Electron Transport in Topological Insulator $\mathrm{Bi}_{2} \mathrm{Se}_{3}$ Nanoribbons, Nanoscale 7 , 16687 (2015).

[58] L. Wu, W.-K. Tse, M. Brahlek, C. M. Morris, R. V. Aguilar, N. Koirala, S. Oh, and N. P. Armitage, High-Resolution Faraday Rotation and Electron-Phonon Coupling in Surface States of the Bulk-Insulating Topological Insulator $\mathrm{Cu}_{0.02} \mathrm{Bi}_{2} \mathrm{Se}_{3}$, Phys. Rev. Lett. 115, 217602 (2015).

[59] T. Liang, Q. Gibson, M. N. Ali, M. Liu, R. Cava, and N. Ong, Ultrahigh Mobility and Giant Magnetoresistance in the Dirac Semimetal $\mathrm{Cd}_{3} \mathrm{As}_{2}$, Nat. Mater. 14, 280 (2015).

[60] T. Champel and V. Mineev, de Haas-van Alphen Effect in Two- and Quasi-Two-Dimensional Metals and Superconductors, Philos. Mag. B 81, 55 (2001).

[61] V. Laukhin, A. Audouard, H. Rakoto, J. Broto, F. Goze, G. Coffe, L. Brossard, J. Redoules, M. Kartsovnik, N. Kushch, L. I. Buravov, A. G. Khomenko, E. B. Yagubskii, S. Askenazy, and P. Pari, Transport Properties and Giant
Shubnikov-de Haas Oscillations in the First Organic Conductor with Metal Complex Anion Containing Selenocyanate Ligand, $(\mathrm{ET})_{2} \mathrm{TlHg}(\mathrm{SeCN})_{4}$, Physica (Amsterdam) 211B, 282 (1995).

[62] S. E. Sebastian, N. Harrison, M. Altarawneh, R. Liang, D. Bonn, W. Hardy, and G. Lonzarich, Chemical Potential Oscillations from Nodal Fermi Surface Pocket in the Underdoped High-Temperature Superconductor $\mathrm{YBa}_{2} \mathrm{Cu}_{3} \mathrm{O}_{6+x}$, Nat. Commun. 2, 471 (2011).

[63] S. G. Sharapov, V. P. Gusynin, and H. Beck, Magnetic Oscillations in Planar Systems with the Dirac-like Spectrum of Quasiparticle Excitations, Phys. Rev. B 69, 075104 (2004).

[64] J. G. Analytis, R. D. McDonald, S. C. Riggs, J.-H. Chu, G. Boebinger, and I. R. Fisher, Two-Dimensional Surface State in the Quantum Limit of a Topological Insulator, Nat. Phys. 6, 960 (2010).

[65] J. Xiong, Y. Luo, Y. H. Khoo, S. Jia, R. J. Cava, and N. P. Ong, High-Field Shubnikov-de Haas Oscillations in the Topological Insulator $\mathrm{Bi}_{2} \mathrm{Te}_{2} \mathrm{Se}$, Phys. Rev. B 86, 045314 (2012).

[66] C. Putzke, L. Malone, S. Badoux, B. Vignolle, D. Vignolles, W. Tabis, P. Walmsley, M. Bird, N. E. Hussey, C. Proust, and Antony Carrington, Inverse Correlation between Quasiparticle Mass and $\mathrm{T}_{c}$ in a Cuprate High- $\mathrm{T}_{c}$ Superconductor, Sci. Adv. 2, e1501657 (2016).

[67] A. A. Taskin and Y. Ando, Berry Phase of Nonideal Dirac Fermions in Topological Insulators, Phys. Rev. B 84, 035301 (2011).

[68] Y. Luo, H. Chen, J. Dai, Z.-A. Xu, and J. D. Thompson, Heavy Surface State in a Possible Topological Kondo Insulator: Magnetothermoelectric Transport on the (011) Plane of $\mathrm{SmB}_{6}$, Phys. Rev. B 91, 075130 (2015).

[69] W. Ruan, C. Ye, M. Guo, F. Chen, X. Chen, G.-M. Zhang, and Y. Wang, Emergence of a Coherent In-Gap State in the $\mathrm{SmB}_{6}$ Kondo Insulator Revealed by Scanning Tunneling Spectroscopy, Phys. Rev. Lett. 112, 136401 (2014).

[70] S. Rößler, T.-H. Jang, D.-J. Kim, L. Tjeng, Z. Fisk, F. Steglich, and S. Wirth, Hybridization Gap and Fano Resonance in $\mathrm{SmB}_{6}$, Proc. Natl. Acad. Sci. U.S.A. 111, 4798 (2014).

[71] L. Jiao, S. Rößler, D. Kim, L. Tjeng, Z. Fisk, F. Steglich, and S. Wirth, Additional Energy Scale in $\mathrm{SmB}_{6}$ at LowTemperature, Nat. Commun. 7, 13762 (2016). 